\title{
Quasinormal ringing of Kerr black holes: The excitation factors
}

\author{
Emanuele Bert:* \\ McDonnell Center for the Space Sciences, Department of Physics, \\ Washington University, St. Louis, Missouri 63130, USA \\ Vitor Cardosd $\dagger$ \\ Dept. of Physics and Astronomy, The University of Mississippi, University, MS 38677-1848, USA
}

(Dated: October 30, 2018)

\begin{abstract}
Distorted black holes radiate gravitational waves. In the so-called ringdown phase radiation is emitted as a discrete set of complex quasinormal frequencies, whose values depend only on the black hole's mass and angular momentum. Ringdown radiation could be detectable with large signal-tonoise ratio by the Laser Interferometer Space Antenna (LISA). If more than one mode is detected, tests of the black hole nature of the source become possible. The detectability of different modes depends on their relative excitation, which in turn depends on the cause of the perturbation (i.e. on the initial data). A "universal", initial data-independent measure of the relative mode excitation is encoded in the poles of the Green's function that propagates small perturbations of the geometry ("excitation factors"). We compute the excitation factors for general-spin perturbations of Kerr black holes. We find that for corotating modes with $l=m$ the excitation factors tend to zero in the extremal limit, and that the contribution of the overtones should be more significant when the black hole is fast rotating. We also present the first analytical calculation of the large-damping asymptotics of the excitation factors for static black holes, including the Schwarzschild and Reissner-Nordström metrics. This is an important step to determine the convergence properties of the quasinormal mode expansion.
\end{abstract}

PACS numbers: 04.70.-s, 04.30.Db, 04.80.Cc, 04.80.Nn

\section{INTRODUCTION}

Distorted black holes emit gravitational radiation as a discrete sum of quasinormal modes (QNMs), damped oscillations whose frequencies and damping times depend only on the black hole's mass and angular momentum. The QNM frequencies scale with the inverse of the black hole's mass. Therefore, the optimal sensitivity bandwidth of each detector determines the mass range that can be detected. The dominant QNM frequency for quadrupolar radiation from a Schwarzschild black hole is $f=1.207 \times 10^{-2}\left(10^{6} M_{\odot} / M\right) \mathrm{Hz}$. As a consequence, the collapse of Population-III stars with mass $M \gtrsim 260 M_{\odot}$ or $25 M_{\odot} \lesssim M \lesssim 40 M_{\odot}$ forms massive black hole remnants that could be detectable by ground-based, high-frequency gravitational wave interferometers such as the advanced Laser Interferometer Gravitational-Wave Observatory (LIGO) [1] and Virgo 2]. The space-based Laser Interferometer Space Antenna (LISA), being sensitive in the frequency band $\sim 10^{-5}-10^{-1} \mathrm{~Hz}$, will detect the gravitational radiation emitted by oscillating black holes of mass $10^{5} M_{\odot} \lesssim M \lesssim 10^{8}-10^{9} M_{\odot}$ with large signal-to-noise ratio (SNR) throughout the observable Universe [3, 4]. With large SNR come precise measurements of the source parameters, and an extraordinary opportunity to study the physics of massive black holes [5].

Since the LISA SNR can be very large, more than one mode could be detected in the ringdown waveform. Such a detection would allow an unprecedented test of the black hole nature of the source. The basic idea is quite simple. In general relativity the complex QNM frequencies form a discrete set $\omega_{l m n}$ classified by three integers: the indices $(l, m)$ come from the separation of the angular dependence of the perturbations, and the index $n$ labels frequencies by the magnitude of their imaginary part (large $n$ means large imaginary part and short damping time). Because of the "no-hair theorem", QNM frequencies depend only on the mass and angular momentum of a Kerr black hole. Roughly speaking, the measurement of one complex frequency (two observables) provides us with a determination of the mass and angular momentum of the black hole, and the measurement of the second frequency allows a consistency check with the general relativistic predictions [ $[4,[5,6,6]$.

\footnotetext{
¥ Also at Centro de Física Computacional, Universidade de Coimbra, P-3004-516 Coimbra, Portugal

*Electronic address: berti@wugrav.wustl.edu

$\dagger$ Electronic address: vcardoso@phy.olemiss.edu
} 
The detectability of different QNMs depends on the relative QNM excitation, which in turn is determined by the cause of the perturbation (i.e. by the initial data). Given a detection, the resolvability of different modes depends on the nature of the multimode ringdown waveform. Two scenarios are possible [4]. If $l \neq l^{\prime}$ or $m \neq m^{\prime}$ (the modes have different angular dependence) the angular scalar product between the modes is zero to a good approximation [8], and the SNR can be expressed as a sum in quadrature of the single-mode SNRs. In [4] we called these multimode ringdown waveforms quasi-orthonormal. If instead $l=l^{\prime}$ and $m=m^{\prime}$, but $n \neq n^{\prime}$ (we look at different overtones with the same angular dependence) the angular scalar product is very close to unity, and mixed terms appear in the SNR calculation and in calculations of parameter estimation accuracy. We may call these waveforms quasi-parallel.

A quantitative estimate of the relative QNM excitation for quasi-orthonormal waveforms will require numerical relativistic simulations and realistic initial data for the merger [9]. However, for quasi-parallel waveforms perturbation theory (combined with a good approximation to the initial data for ringdown) can provide useful information on the relative excitation of different overtones. In this paper, using perturbation theory, we develop tools to estimate the relative excitation of different overtones of Kerr black holes for general classes of initial data. We also try to gain some theoretical insight into the initial-data dependence of the results for realistic mergers, considering simple classes of initial data (such as localized spikes and gaussians) as model problems.

Our work is the first application to gravitational perturbations of Kerr black holes of a theoretical framework developed over the years by different authors. Following the pioneering numerical analysis by Vishveshwara [10], who studied the scattering of gaussian wave pulses on the Schwarzschild background, a number of studies investigated the analytical structure of the Green's function that propagates small perturbations in black hole geometries. Our own analysis is based on the formalism developed by Leaver in the eighties 11, 12, 13], but the Green's function in the Schwarzschild background has been studied by many authors 14, 15, 16, 17, 18|. More recently Glampedakis and Andersson extended the analysis to scalar perturbations of Kerr black holes [19, 20]. Here we carry out the first study of general-spin perturbations of Kerr black holes, including the physically most important case of gravitational perturbations.

The main result emerging from $[11,12,13,14,15,16,17,18,19,20]$ is that a "universal" (initial-data independent) measure of the relative QNM excitation is encoded in the poles of the Green's function that propagates small perturbations of the geometry. These "universal" quasinormal excitation factors (QNEFs) are defined in Eq. (3.8) below. They depend only on the Kerr geometry, not on the details of the perturbation. When combined with a knowledge of the initial data they can be used to compute the so-called quasinormal excitation coefficients defined in Eq. (3.9), which are a concrete measure of the QNM content of a waveform.

The paper is organized as follows. In Sec. [II to develop some physical intuition, we consider a very simple physical system: a vibrating string with fixed ends. This part provides a useful pedagogical introduction to the QNM excitation problem, and may be skipped by readers familiar with the topic. We consider the Green's function solution of the vibrating string equation for generic initial data, identify the normal modes of the system as poles of the Green's function and show the importance of initial data to determine the excitation of the modes. In Sec. III we show that many features of the vibrating string problem carry over to black hole perturbation theory, stressing the main differences between normal mode expansions and QNM expansions. We also anticipate some results on the convergence of the QNEFs, which are presented in more detail in Appendix $\mathrm{A}$ In Sec. IV we outline our calculation of the QNEFs, present the numerical results and compute the response of a Kerr black hole to localized and gaussian initial data. Technical details, as well as a discussion of the numerical accuracy of our calculations, are relegated to Appendix B. Appendix [C clarifies the relation between the Teukolsky and Sasaki-Nakamura (SN) formulations of the Kerr perturbation equations, and between the corresponding QNEFs.

\section{A PEDAGOGICAL EXAMPLE: A VIBRATING STRING}

Some key features of the black hole perturbation problem we address in this paper are exemplified by a very simple system: a vibrating string with fixed ends. To simplify the mathematics we pick units so that the velocity of the waves in the string $c=1$, and consider a string of length $\pi$. Then any disturbance of the string obeys the wave equation

$$
\frac{\partial^{2} u}{\partial t^{2}}=\frac{\partial^{2} u}{\partial x^{2}} \quad \text { on } \quad 0 \leq x \leq \pi
$$

with $u(t, 0)=u(t, \pi)=0$. The general solution of this problem is easily verified to be

$$
u(t, x)=\sum_{n=1}^{\infty}\left(\bar{C}_{n} \cos n t+\bar{C}_{n}^{\prime} \sin n t\right) \sin (n x),
$$


where we used an overbar to avoid confusion with the quasinormal excitation coefficients $C_{n}$, as defined in Eq. (3.9) below. In Fourier language we say the general solution is a superposition of normal modes with sinusoidal dependence on $x$ and $t$, labeled by an integer $n$. Each mode has frequency $\omega=n$.

For the general solution to be useful we must determine the constants $\bar{C}_{n}$ and $\bar{C}_{n}^{\prime}$, that is, we must determine the contribution of each individual mode. This contribution can easily be computed once we are given initial data, namely the initial configuration $u(0, x) \equiv u_{0}(x)$ and velocity profile $\partial_{t} u(0, x) \equiv v_{0}(x)$ of the string. Indeed, consider (2.2) and its first derivative, both evaluated at $t=0$. Multiplying both sides by $\sin n x$ and integrating on $(0, \pi)$ we get

$$
\bar{C}_{n}=\frac{2}{\pi} \int_{0}^{\pi} u_{0}(x) \sin n x d x, \quad \bar{C}_{n}^{\prime}=\frac{2}{n \pi} \int_{0}^{\pi} v_{0}(x) \sin n x d x,
$$

which completely specifies the solution.

In more general situations it is not possible to find closed-form elementary solutions satisfying some given boundary conditions (in our vibrating string example, the fixed ends condition). However, an elegant formal solution can be obtained using Green's functions. Let us consider a slight generalization of Eq. (2.1):

$$
\frac{\partial^{2} u}{\partial x^{2}}-\frac{\partial^{2} u}{\partial t^{2}}-V(x) u=\mathcal{S}
$$

where we introduced a potential $V(x)$ and a source $\mathcal{S}$ representing, say, external forces acting on the system (for a free vibrating string $V(x)=\mathcal{S}=0)$. We define the Laplace transform of $u(t, x)$ as $^{1}$

$$
\mathcal{L} u(t, x) \equiv \hat{u}(\omega, x)=\int_{t_{0}}^{\infty} u(t, x) e^{i \omega t} d t
$$

In terms of the Laplace transform, the original field can be written as

$$
u(t, x)=\frac{1}{2 \pi} \int_{-\infty+i c}^{\infty+i c} \hat{u}(\omega, x) e^{-i \omega t} d \omega .
$$

Using the elementary property $\mathcal{L}\left[\frac{\partial u(t, x)}{\partial t}\right]=-i \omega \mathcal{L} u-e^{i \omega t_{0}} u\left(x, t_{0}\right)$, the Laplace transformation of (2.4) leads to

$$
\frac{\partial^{2} \hat{u}}{\partial x^{2}}+\left[\omega^{2}-V(x)\right] \hat{u}=I(\omega, x)
$$

where

$$
I(\omega, x)=e^{i \omega t_{0}}\left[i \omega u(t, x)-\frac{\partial u(t, x)}{\partial t}\right]_{t=t_{0}}+\hat{\mathcal{S}}
$$

This equation is formally solved with the use of a Green's function $G\left(x, x^{\prime}\right)$ such that

$$
\frac{\partial^{2} \hat{u}}{\partial x^{2}}+\left[\omega^{2}-V(x)\right] G\left(x, x^{\prime}\right)=\delta\left(x-x^{\prime}\right) .
$$

In terms of the Green's function the solution is simply given by

$$
\hat{u}=\int I\left(\omega, x^{\prime}\right) G\left(x, x^{\prime}\right) d x^{\prime} .
$$

Suppose we know the Green's function. Then the previous equation shows that, given $I\left(\omega, x^{\prime}\right)$ (which means, in the absence of external forces, given initial data) we can determine, at least in principle, the solution.

There is a general prescription to construct the Green's function [21]. Find two linearly independent solutions of the homogeneous equation, say $\hat{u}_{1}(\omega, x)$ and $\hat{u}_{2}(\omega, x)$, each satisfying one of the required boundary conditions: for the vibrating string these solutions would be such that $\hat{u}_{1}(\omega, 0)=0, \hat{u}_{2}(\omega, \pi)=0$. The Green's function is then

$$
G\left(x, x^{\prime}\right)=\frac{1}{W}\left\{\begin{array}{l}
\hat{u}_{1}(x) \hat{u}_{2}\left(x^{\prime}\right) \text { if } x \leq x^{\prime}, \\
\hat{u}_{1}\left(x^{\prime}\right) \hat{u}_{2}(x) \text { if } x^{\prime} \leq x,
\end{array}\right.
$$

\footnotetext{
1 The usual Laplace variable $s=-i \omega$. We prefer to use $\omega$ for notational consistency with previous work by Leaver [13] and Andersson [17]. Our transform is well defined as long as $\operatorname{Im}(\omega) \geq c$.
} 
where we defined the Wronskian between the two solutions

$$
W=\frac{\partial \hat{u}_{1}}{\partial x} \hat{u}_{2}-\hat{u}_{1} \frac{\partial \hat{u}_{2}}{\partial x},
$$

which for equations of the type (2.7) is a constant. For the vibrating string the homogeneous solutions are elementary functions: $\hat{u}_{1}=\sin \omega x, \hat{u}_{2}=\sin \omega(x-\pi)$, and the Green's function

$$
G\left(x, x^{\prime}\right)= \begin{cases}-\frac{\sin \omega x \sin \omega\left(x^{\prime}-\pi\right)}{\omega \sin \omega \pi} & \text { if } x \leq x^{\prime}, \\ -\frac{\sin \omega x^{\prime} \sin \omega(x-\pi)}{\omega \sin \omega \pi} & \text { if } x^{\prime} \leq x .\end{cases}
$$

Notice that the Wronskian $W=-\omega \sin \omega \pi$ is zero at $\omega=n$ with $n$ integer, that is, at the normal frequencies of the system. Near the poles $\omega=n$ we have $W \simeq-[n \pi \cos (n \pi)](\omega-n)$. Corresponding to zeros of the Wronskian (which are also poles of the Green's function: see below) the two solutions $\hat{u}_{1}$ and $\hat{u}_{2}$ are no longer independent: they satisfy both boundary conditions simultaneously. In fact, setting $\omega=n$ in (2.13) we can see that $\hat{u}_{1}$ and $\hat{u}_{2}$ coincide, and correspond to the normal modes of the system. Using (2.6), (2.8) and (2.10) and setting for simplicity $t_{0}=\mathcal{S}=0$ we get

$$
u(t, x)=\frac{1}{2 \pi} \int d x^{\prime} d \omega\left[i \omega u_{0}\left(x^{\prime}\right)-v_{0}\left(x^{\prime}\right)\right] G\left(x, x^{\prime}\right) e^{-i \omega t}
$$

The $\omega$-integral can be performed by closing the contour of integration. We choose the contour depicted in Fig. 1

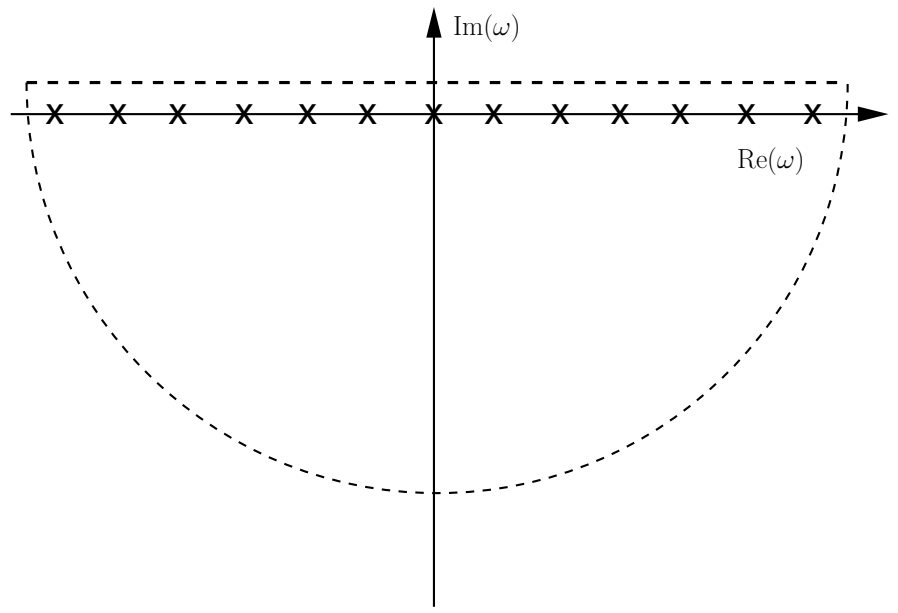

FIG. 1: Integration contour for the vibrating string problem. Crosses mark zeros of the Wronskian $W$, corresponding to the normal frequencies of the system.

If $\hat{u}_{1}(x), \hat{u}_{2}(x)$ are analytic and have no essential singularities inside the contour ${ }^{2}$, the poles of the Green's function are all due to zeros of the Wronskian. In this way we get for the integral in (2.14),

$$
\begin{aligned}
u(t, x)= & \frac{1}{2 \pi} \int d x^{\prime} d \omega\left[i \omega u_{0}\left(x^{\prime}\right)-v_{0}\left(x^{\prime}\right)\right] G\left(x, x^{\prime}\right) e^{-i \omega t} \\
= & -i \sum_{n} \frac{1}{n \pi}\left\{\int d x^{\prime} \sin n x^{\prime}\left[i n u_{0}\left(x^{\prime}\right)-v_{0}\left(x^{\prime}\right)\right]\right\} e^{-i n t} \sin n x \\
& +\frac{1}{2 \pi} \int_{H C} d \omega \int d x^{\prime}\left[i \omega u_{0}\left(x^{\prime}\right)-v_{0}\left(x^{\prime}\right)\right] G\left(x, x^{\prime}\right) e^{-i \omega t},
\end{aligned}
$$

\footnotetext{
${ }^{2}$ Both conditions are met in the case of a vibrating string, but both are violated when we deal with black hole QNMs. For black holes, the Green's function essential singularity at the origin gives rise to tails, and the integral over the half-circle at infinity is responsible for the early-time response of the black hole. See discussion after Eq. 3.6.
} 
where "HC" in the second term means that the integration should be performed along the half-circle. Not surprisingly, taking the real part of the right hand side we recover the result (2.2) with expansion coefficients given by (2.3).

The bottom line of this discussion is that the general solution depends crucially on two elements: (i) the residues of the Green's function evaluated at the poles (that is, at the normal frequencies); (ii) the function $I(\omega, x)$, which (in the absence of initial forces) is nothing but the initial data. By inspection, the net result for the field can be expressed as a sum of the form

$$
u(t, x) \propto \sum_{n} \frac{J_{n}(t, x)}{\partial_{\omega} W} u_{n}\left(\omega_{n}, x\right) e^{-i \omega_{n} t}
$$

where $\omega_{n}=n$ is a normal frequency, $J_{n}(t, x)=\int d x^{\prime} I\left(\omega_{n}, x^{\prime}\right) u_{n}\left(\omega_{n}, x^{\prime}\right)$ and $u_{n}\left(\omega_{n}, x\right)=\sin n x$ is a normal mode wavefunction (that is, any of the homogeneous solutions evaluated at the normal frequency $\omega_{n}=n$ ). We will see below that a similar result holds for the ringdown of Kerr black holes.

\section{A. Effect of initial data}

In the previous section we pointed out that initial data play a crucial role to determine the excitation of the normal modes of a system. For illustration, below we consider three simple examples that will be useful in the following to understand, by analogy, the initial-data dependence of the excitation of a Kerr black hole.

Suppose first that we have an (initially stationary) plucked string: the string's initial profile is a triangle of height $h$ with a vertex at $x^{S}$, i.e. $v_{0}(x)=0$ and

$$
u_{0}(x)= \begin{cases}\frac{x h}{x^{S}} & \text { if } 0 \leq x \leq x^{S} \\ \frac{h(\pi-x)}{\pi-x^{S}} & \text { if } x^{S} \leq x \leq \pi .\end{cases}
$$

Stationarity of the initial data implies that $\bar{C}_{n}^{\prime}=0$, so that

$$
u(t, x)=\sum_{n=1}^{\infty} \bar{C}_{n} \cos n t \sin n x, \quad \bar{C}_{n}=\frac{2 h}{n^{2}} \frac{\sin n x^{S}}{x^{S}\left(\pi-x^{S}\right)}
$$

Notice that modes having a node at $x^{S}$, where the string is plucked, are not excited $\left(\bar{C}_{n}=0\right)$. Notice also that the "excitation factors" $\bar{C}_{n}$ decrease as $1 / n^{2}$.

As a second example take stationary, localized " $\delta$-function" initial data of the form

$$
u_{0}(x)=\delta\left(x-x^{S}\right), \quad v_{0}(x)=0 .
$$

The excitation factors are trivially computed:

$$
\bar{C}_{n}=\frac{2}{\pi} \sin \left(n x^{S}\right)
$$

It is apparent that all modes are excited to a comparable amplitude except for modes with a node at the plucking point, which are not excited at all.

Our third and last example are stationary, gaussian initial data:

$$
u_{0}(x)=e^{-b\left(x-x^{S}\right)^{2}}, \quad v_{0}(x)=0 .
$$

For large $b$ the gaussian is strongly peaked at $x=x^{S}$, in which case the contribution to the integral outside of $(0, \pi)$ can be ignored and we have

$$
\bar{C}_{n}=\frac{2}{\pi} \int_{0}^{\pi} u_{0}(x) \sin n x d x \simeq \frac{2}{\pi} \int_{-\infty}^{\infty} e^{-b\left(x-x^{S}\right)^{2}} \sin n x d x=\frac{2 \sin \left(n x^{S}\right)}{\sqrt{\pi b}} e^{-n^{2} /(4 b)}
$$

Therefore a mode with given $n$ is maximally excited when the width of the gaussian satisfies the condition $b=n^{2} / 2$. A similar result will be seen to hold for gaussians exciting Kerr black holes.

The basic lesson we learn from these examples is that the excitation of a system is very sensitive to the initial data. More specifically, whether a given mode is excited or not depends strongly on the point where we excite the system ("pluck the string"). 


\section{OSCILLATING BLACK HOLES}

In the SN formalism [22, 23, 24, 25], perturbations of a Kerr black hole induced by a spin- $s$ field are described by a single function $X^{(s)}(t, r)$ whose Laplace transform satisfies

$$
\frac{d^{2} \hat{X}^{(s)}(\omega, r)}{d r_{*}^{2}}+V_{S N} \hat{X}^{(s)}(\omega, r)=I(\omega, r),
$$

where the effective potential $V_{S N}$ depends both on the radial coordinate and on the frequency. The function $I(\omega, r)$ is a linear combination of the SN function $X^{(s)}\left(t_{0}, r\right)$ and its time derivative $\dot{X}^{(s)}\left(t_{0}, r\right)$ at time $t_{0}$ (see below for the explicit expression for scalar perturbations). The tortoise coordinate $r_{*}$ is defined by the condition

$$
\frac{d r_{*}}{d r}=\frac{r^{2}+a^{2}}{\Delta},
$$

ranging from $-\infty$ (the location of the event horizon) to $+\infty$ (spatial infinity). We use Boyer-Lindquist coordinates and follow Leaver's choice of units, setting $G=c=2 M=1$. In Leaver's units the angular momentum per unit mass $a$ is such that $0 \leq a \leq M=1 / 2$, and the horizon function $\Delta=r^{2}-r+a^{2}$. Sometimes we will present our results in terms of the more familiar dimensionless angular momentum $j=2 a$, such that $0 \leq j \leq 1$. The class of problems that fit in this description include any massless field in the Kerr geometry, including gravitational, electromagnetic and scalar fields [26].

The main difference with the vibrating string example is that our system is not conservative: waves can escape to infinity. For this reason an expansion in normal modes is not possible (see [13, 15, 27] for extensive discussions of this point). Wave propagation is also complicated by backscattering off the background curvature, which is responsible for tail effects [28]. Despite these complications it can be shown that the poles of the Green's function (now located at complex frequencies corresponding to the QNMs) still play an important role in the evolution.

The QNM contribution can be isolated from other features of the signal, such as the late-time tail, using the Green's function technique [13, 17]. First one defines a solution of the homogeneous equation having the correct behavior at the horizon (only in-going waves),

$$
\begin{aligned}
& \lim _{r \rightarrow r_{+}} \hat{X}_{r_{+}}^{(s)} \sim e^{-i(\omega-m \Omega) r_{*}}, \\
& \lim _{r \rightarrow \infty} \hat{X}_{r_{+}}^{(s)} \sim A_{\text {in }}(\omega) e^{-i \omega r_{*}}+A_{\text {out }}(\omega) e^{i \omega r_{*}},
\end{aligned}
$$

where $r_{ \pm}=\left[1 \pm\left(1-4 a^{2}\right)^{1 / 2}\right] / 2$, and a second solution $\hat{X}_{\infty_{+}}^{(s)}$ behaving as $e^{i \omega r_{*}}$ for large values of $r$. Since the Wronskian $W=2 i \omega A_{\text {in }}$ we can express the general solution as [17]

$$
\hat{X}^{(s)}(\omega, r)=\hat{X}_{\infty_{+}}^{(s)} \int_{-\infty}^{r_{*}} \frac{I(\omega, r) \hat{X}_{r_{+}}^{(s)}}{2 i \omega A_{\mathrm{in}}} d r_{*}^{\prime}+\hat{X}_{r_{+}}^{(s)} \int_{r_{*}}^{\infty} \frac{I(\omega, r) \hat{X}_{\infty_{+}}^{(s)}}{2 i \omega A_{\mathrm{in}}} d r_{*}^{\prime} .
$$

To proceed we make the astrophysically reasonable assumption that the observer is located far away from the black hole. If the initial data have compact support and this support is entirely located closer to the black hole with respect to the observer (this is basically a "no-incoming radiation from infinity" condition), a good approximation will be

$$
\hat{X}^{(s)}(\omega, r) \simeq \frac{e^{i \omega r_{*}}}{2 i \omega A_{\text {in }}} \int_{-\infty}^{\infty} I(\omega, r) \hat{X}_{r_{+}}^{(s)} d r_{*}^{\prime}
$$

As explained in [13, 17, 29], when we invert this expression to get the solution in the time domain we get (as in the vibrating string case) a contribution from the poles of the Green's function. This contribution can again be isolated by closing the path of integration, as was done in Eq. (2.15). An important difference that distinguishes black hole spacetimes is that there is now an essential singularity at $\omega=0$ and a branch cut extending from the singularity to $-i \infty[29,30,31]$. To prevent the essential singularity from lying inside the integration contour we must modify slightly the contour of Fig. 1. We place a branch cut along the negative imaginary- $\omega$ axis and split the half circle at $|\omega| \rightarrow \infty$ into two quarter circles. The new contour is shown in Fig. 2. The poles in the complex frequency plane are the zeros of $A_{\mathrm{in}}$ : they correspond to perturbations satisfying both in-going wave conditions at the horizon and out-going wave conditions at infinity, that is (by definition) to QNMs. QNM frequencies have negative imaginary part. Since we assume a Fourier dependence of the form $X^{(s)}(t, r) \sim e^{-i \omega t}$ this means that there are no exponentially growing modes. There is an infinity of QNMs, which are usually sorted by the magnitude of their imaginary part and labelled by an integer $n$. 


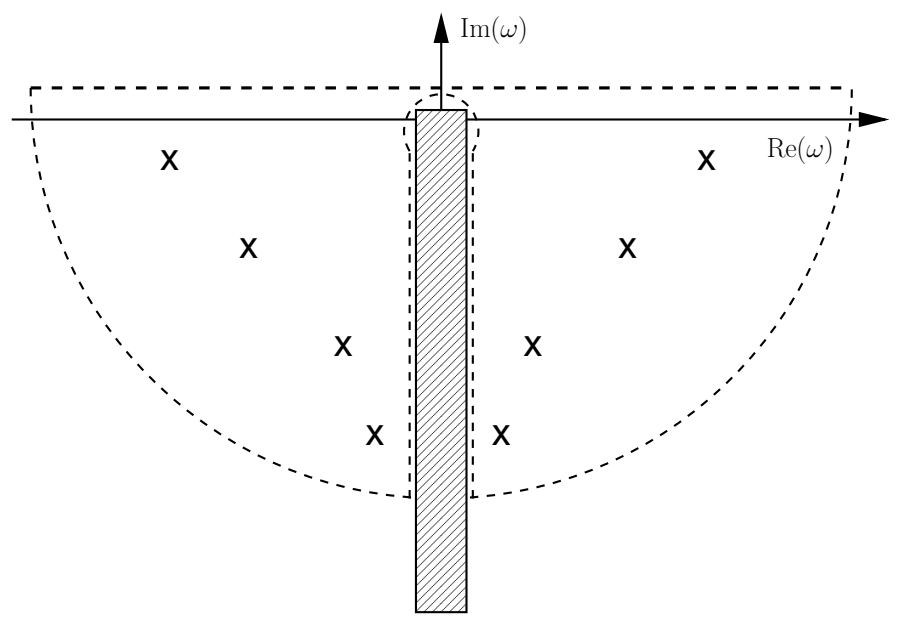

FIG. 2: Integration contour to invert Eq. (3.6). The shaded area is the branch cut and crosses mark zeros of the Wronskian $W$ (the QNM frequencies).

Extracting the QNM contribution ${ }^{3}$ to the radiated wave we get

$$
\begin{aligned}
X^{(s)}(t, r) & =-\operatorname{Re}\left[\sum_{n} B_{n} e^{-i \omega_{n}\left(t-r_{*}\right)} \int_{-\infty}^{\infty} \frac{I(\omega, r) \hat{X}_{r_{+}}^{(s)}}{A_{\mathrm{out}}} d r_{*}^{\prime}\right] \\
& =-\operatorname{Re}\left[\sum_{n} C_{n} e^{-i \omega_{n}\left(t-r_{*}\right)}\right]
\end{aligned}
$$

where the sum is over all poles in the complex plane and the quasinormal excitation factors (QNEFs) $B_{n}$ are defined as

$$
B_{n}=\left.\frac{A_{\text {out }}}{2 \omega \alpha_{n}} \equiv \frac{A_{\text {out }}}{2 \omega}\left(\frac{d A_{\text {in }}}{d \omega}\right)^{-1}\right|_{\omega=\omega_{n}} .
$$

Here $\alpha_{n}$ is a commonly used notation for the derivative of $A_{\text {in }}$ at the QNM frequency. The quasinormal excitation coefficients $C_{n}$ can be computed as

$$
C_{n}=B_{n} \int_{-\infty}^{\infty} \frac{I(\omega, r) \hat{X}_{r_{+}}^{(s)}}{A_{\text {out }}} d r_{*}^{\prime}
$$

whenever the integral on the right hand side, which must be evaluated at the QNM frequency $\omega=\omega_{n}$, is convergent. In general the QNM frequencies $\omega_{n}$, the $B_{n}$ 's and the $C_{n}$ 's (as well as the wavefunction) depend on $(l, m)$ and the spin of the perturbing field $s$, but to simplify the notation we will omit this dependence whenever there is no risk of confusion.

By definition of the QNM frequencies, $\hat{X}_{r_{+}}^{(s)} \sim A_{\text {out }} e^{i \omega r_{*}}$ as $r_{*} \rightarrow \infty$ at $\omega=\omega_{n}$ : in this sense the above integral is "normalized". For source terms $I(\omega, r)$ that are zero outside some finite range of $r$, or have sufficiently rapid exponential decay as $\left|r_{*}\right| \rightarrow \infty$, the integral is also convergent. For other classes of initial data the integral as evaluated on the real line is, in general, divergent. This is a major difference with respect to ordinary normal mode expansions. The normal modes of closed mechanical systems are Sturm-Liouville eigenfunctions of the wave equation, and their excitation coefficients are weighted integrals of the source term over the mode. Since the Sturm-Liouville eigenfunctions are always bounded, the integrals always converge. For QNM expansions a meaningful definition of

\footnotetext{
3 There are also other contributions to the signal. The essential singularity at the origin $(\omega=0)$ gives rise to the tail of the time evolved wavefunction; the integral over the quarter circles at infinite frequency produces the early time response of the black hole. Since our main focus is the QNM contribution we discard these two terms in the integral. For more details we refer the reader to [13, 29, 31].
} 
the integral for general source terms requires more care, and the introduction of an analytical continuation procedure [13, 14].

Equation (3.7) is one of the main results we will use throughout the rest of the paper. Once we specify initial data, the QNEFs allow the determination of the QNM content of a signal. QNEFs have long been known for scalar, electromagnetic and gravitational perturbations of Schwarzschild black holes [13, 14, 17]. More recently there have been attempts to extend those calculations to scalar perturbations of Kerr black holes [19, 20]. Electromagnetic and (most importantly for gravitational-wave phenomenology) gravitational perturbations of Kerr black holes have not been dealt with so far. One purpose of the present work is to fill this gap. Before turning to an explicit calculation of the $B_{n}$ 's we will provide the explicit form of the function $I(\omega, r)$ for $s=0$. We will also address two important conceptual issues: the time-shift problem and the convergence of the QNM expansion.

\section{A. Initial data for scalar perturbations}

For a scalar field [19] the function $I(\omega, r)$ is given by

$$
I(\omega, r)=e^{i \omega t_{0}}\left\{i\left[\omega-\frac{2 a m r+\omega a^{2} \Delta \gamma_{l m}}{\left(r^{2}+a^{2}\right)^{2}}\right] X^{(0)}\left(t_{0}, r\right)-\left[1-\frac{a^{2} \Delta \gamma_{l m}}{\left(r^{2}+a^{2}\right)^{2}}\right] \dot{X}^{(0)}\left(t_{0}, r\right)\right\},
$$

where $\gamma_{l m}$ is a number depending on the multipole of the field under study:

$$
\gamma_{l m}=2 \pi \int_{0}^{\pi} S_{l m}^{*}(a \omega, \theta) S_{l m}(a \omega, \theta) \sin ^{3} \theta d \theta
$$

Here the $S_{l m}$ 's are scalar $(s=0)$ spin-weighted spheroidal harmonics [8]. We require them to satisfy the normalization condition $2 \pi \int_{0}^{\pi} S_{l m}^{*}(a \omega, \theta) S_{l m}(a \omega, \theta) \sin \theta d \theta=1$ (notice that this normalization differs by a factor $2 \pi$ from that adopted in [19]). Even though these functions depend on $a \omega$, in [4] we showed that in some cases (for example, when we are dealing with slowly damped QNMs) this dependence can safely be neglected. When $a \omega=0$ scalar spheroidal harmonics reduce to the usual spherical harmonics, and the integral can be computed analytically with the result

$$
\gamma_{l m}=\frac{2\left(l^{2}+l-1+m^{2}\right)}{(2 l-1)(3+2 l)} .
$$

For the calculations in this paper, the analytic formula is extremely accurate even when $a \omega \neq 0$. For example, for a near-extremal black hole with $j=0.98$ a numerical evaluation of the integral at the fundamental scalar QNM frequency yields $\gamma_{22}=0.854139$, in excellent agreement with the analytic prediction $\gamma_{22}=6 / 7 \simeq 0.857143$. Results for other slowly-damped modes are similar.

In the following of the paper we will focus, for simplicity, on static initial data. For large $r$ Eq. (3.10) reduces to

$$
I(\omega, r) \sim i \omega e^{i \omega t_{0}} X^{(0)}\left(t_{0}, r\right)
$$

whereas for $r$ very near the horizon we get

$$
I(\omega, r)=i e^{i \omega t_{0}}(\omega-2 m \Omega) X^{(0)}\left(t_{0}, r\right),
$$

where $\Omega=a / r_{+}$is the angular velocity of the horizon.

\section{B. The time-shift problem}

Taken at face value Eq. (3.7) is troublesome, because the response diverges (exponentially) for early times. The well-known fact that ringdown waveforms only make sense for certain values of $t$ is usually referred to as the "time-shift problem" [14, 16, 18]. For illustration, consider Eq. (3.7) for a Schwarzschild black hole with static initial data:

$$
X^{(s)}(t, r)=-\operatorname{Re}\left[\sum_{n=0}^{\infty} B_{n} e^{-i \omega_{n}\left(t-r_{*}-t_{0}\right)} \int_{-\infty}^{\infty} \frac{i \omega_{n} X^{(s)}\left(t_{0}, r\right) \hat{X}_{r_{+}}^{(s)}}{A_{\text {out }}} d r_{*}^{\prime}\right] .
$$

The wave field $X^{(s)}(t, r)$ at some observation point $r$ and time $t$ depends exponentially on the (arbitrary) choice of $t_{0}$, which does not make much physical sense. Suppose in addition that the initial data consist of a narrow pulse, 
say for simplicity a $\delta$-function: $X^{(s)}\left(t_{0}, r\right)=\delta\left(r-r^{S}\right)$. Far away from the black hole $\hat{X}_{r_{+}}^{(s)} \sim A_{\text {out }} e^{i \omega_{n} r_{*}}$, and the response of the black hole increases exponentially with $r^{S}$. This is against physical intuition: according to our Fourier convention stable oscillations have $\operatorname{Im}(\omega)<0$, so a small bump in initial data far from the black hole seems to excite much more ringing than a huge bump in the strong-field region around the horizon.

The way out of this problem [14, 18] is to realize that ringdown only starts after the initial data (that for simplicity are usually assumed to have compact support and be located in the far zone, $r^{S} \gg 1$ ) reach the potential barrier, where ringdown originates [10], and the perturbation travels back to the observer. Suppose the observer is sitting at some large $r_{*}$ and an impulse localized at $r_{*}=r_{*}^{S}$ falls into the black hole at time $t_{0}$. The Regge-Wheeler "potential barrier" has a location depending on the mode under consideration, and the very notion of potential barrier becomes fuzzy for Kerr black holes. A reasonable estimate is to assume that the "barrier" is located close to $r_{*} \sim 0$, and to avoid complications we will take this estimate as our fiducial value. In geometrical units, based on the above discussion, the starting time for the ringdown signal will be approximately

$$
t_{\mathrm{start}}=\left|r_{*}^{S}\right|+r_{*}+t_{0} .
$$

For the QNM expansion to make sense, the observation time $t$ must always be chosen so that $t \geq t_{\text {start }}$. This choice partially gets rid of the time-shift problem, in the sense that $t_{0}$ and $r_{*}^{S}$ do not appear explicitly in the waveform.

A more rigorous approach should also take into account the contribution of the prompt response (the analogous of light cone propagation in flat space) and the matching between these two phases. Ref. 18] explores some aspects of this problem, computing the large-frequency contribution to the Green's function (large frequency is roughly equivalent to early times). The final result does not significantly alter our "simple-minded" scenario. We should also mention that our prescription to solve the time-shift problem works reasonably well only for sharply localized initial data, like narrow gaussians. If the initial data have a significant spread in the radial direction the starting time $t_{\text {start }}$ is ill-defined and we must resort to a more detailed analysis, based perhaps on time-dependent excitation coefficients [18]. This problem deserves further investigation.

\section{Convergence of the QNM expansion}

The convergence of the QNM series has been studied in some special important cases by Leaver [13]. In particular he pointed out that the sum can only be expected to converge at times $t$ such that ringdown dominates (see the above discussion of the time-shift problem), and that the ultimate convergence of the series depends on the large- $n$ asymptotics of the QNEFs. The convergence problem was revisited numerically by Andersson [18] computing the $B_{n}$ 's up to $n \sim 200$ for scalar perturbations. His calculation shows that their magnitude decreases monotonically for large $n$. From (3.15) it follows that, if the initial data are localized at $r_{*}=r_{*}^{S} \gg 1$, the ratio of two consecutive terms behaves like $\left(B_{n+1} / B_{n}\right) e^{-i\left(\omega_{n+1}-\omega_{n}\right)\left(t-r_{*}-r_{*}^{S}\right)}$. Asymptotically, $\omega_{n+1} \simeq \omega_{n}-i / 2$ for large $n$. In Appendix A we determine analytically, for the first time, the asymptotic behavior of the QNEFs for a number of static black hole spacetimes. Our most interesting result is that the large- $n$ behavior of scalar and gravitational perturbations of a Schwarzschild black hole is

$$
B_{n}^{(0)}=B_{n}^{(-2)}=-\frac{i}{3[ \pm \log 3-(2 n+1) \pi i]}, \quad(n \rightarrow \infty),
$$

in good agreement with numerical calculations (cf. Figure 2 in Ref. [18]). This implies that $\left|B_{n+1} / B_{n}\right| \rightarrow 1$ in the same limit, so the ratio of consecutive terms is of order $\exp \left[-\left(t-r_{*}-r_{*}^{S}\right) / 2\right]$ and the sum will converge for $\left(t-r_{*}-r_{*}^{S}\right) \gg 0$. This is precisely the kind of convergence we should expect from a QNM expansion.

To prove more rigorously the convergence of the expansion (3.7) we still need two ingredients. The first is the convergence of the integral in (3.9). For generic initial data this integral diverges on the real line. The divergence can be cured by analytic continuation, evaluating the integral as a contour integral (for details see [13, 14]). Secondly, the fact that the QNEFs $B_{n} \sim 1 / n$ for large $n$ does not imply convergence of the corresponding quasinormal excitation coefficients $C_{n}$. A proof of convergence would require the calculation of the integral over initial data and a knowledge (or an estimate of) its $n$-dependence for large $n$.

Having discussed these basic properties of QNM expansions, in the following of the paper we turn to an explicit calculation of the $j$-dependence of the QNEFs for different QNMs and for different spins of the perturbing field. 


\section{QUASINORMAL EXCITATION FACTORS IN THE KERR BACKGROUND}

\section{A. Formalism}

Perturbations in the Kerr geometry can be studied using both the Teukolsky [32] and SN [23] formalisms. There are two main advantages in using the SN formalism: (i) the potential and source in the SN wave equation are short ranged and (ii) in the limit $a \rightarrow 0$ the SN wave equation reduces to the Regge-Wheeler equation, describing axial perturbations of a Schwarzschild black hole.

The QNEFs $B_{n}$ depend, by their own definition (3.8), on the amplitude of ingoing and outgoing waves at infinity. Even in the Schwarzschild limit, despite the isospectrality between axial and polar perturbations, the Regge-Wheeler and Zerilli QNEFs differ by a proportionality constant. Denoting quantities in the Zerilli equation by a plus (meaning even-parity), the two are related in a simple way [13]:

$$
A_{\text {out }}^{+}=\frac{(l-1) l(l+1)(l+2)+6 i \omega}{(l-1) l(l+1)(l+2)-6 i \omega} A_{\text {out }}, \quad B_{n}^{+}=\frac{(l-1) l(l+1)(l+2)+6 i \omega}{(l-1) l(l+1)(l+2)-6 i \omega} B_{n} .
$$

Similarly, the SN QNEFs for spin-s perturbations $B^{(s)}$ will differ from the Teukolsky QNEFs (that we will denote by a subscript $\left.T, B_{\mathrm{T}}^{(s)}\right)$ by a proportionality constant. In Appendix $\mathrm{C}$ we derive the following relations:

$$
B_{\mathrm{T}}^{(0)}=B^{(0)}, \quad B_{\mathrm{T}}^{(-1)}=-\frac{4 \omega^{2}}{2 a m \omega-A_{l m}-a^{2} \omega^{2}} B^{(-1)}, \quad B_{\mathrm{T}}^{(-2)}=\frac{16 \omega^{4}}{\lambda(\lambda+2)-6 i \omega-12 a \omega(a \omega-m)} B^{(-2)} .
$$

with $\lambda \equiv A_{l m}+(a \omega)^{2}-2 a m \omega$.

Our calculations in this paper rely heavily on the formalism developed by Leaver [11, 12, 13], which refers to the Teukolsky formulation of the Kerr perturbation equations. In this Section we only outline the basic steps of the calculation, relegating details to Appendix B. Expanding a spin-s field $\psi(t, r, \theta, \phi)$ as

$$
\psi(t, r, \theta, \phi)=\frac{1}{2 \pi} \int e^{-i \omega t} \sum_{l=|s|}^{\infty} \sum_{m=-l}^{l} e^{i m \phi} S_{l m}(\theta) R_{l m}(r) d \omega,
$$

we get ordinary differential equations for $S_{l m}$ and $R_{l m}$ [32]:

$$
\begin{aligned}
& \partial_{u}\left[\left(1-u^{2}\right) \partial_{u} S_{l m}\right]+\left(a^{2} \omega^{2} u^{2}-2 a \omega s u+s+A_{l m}-\frac{(m+s u)^{2}}{1-u^{2}}\right) S_{l m}=0, \\
& \Delta \partial_{r}^{2} R_{l m}+(s+1)(2 r-1) \partial_{r} R_{l m}+V R_{l m}=0,
\end{aligned}
$$

where $u \equiv \cos \theta$ and

$$
V=2 i s \omega r-a^{2} \omega^{2}-A_{l m}+\frac{1}{\Delta}\left[\left(r^{2}+a^{2}\right)^{2} \omega^{2}-2 a m \omega r+a^{2} m^{2}+i s\left(a m(2 r-1)-\omega\left(r^{2}-a^{2}\right)\right)\right] .
$$

The field spin $s=0,-1,-2$ for scalar, electromagnetic and gravitational perturbations, respectively. The separation constant $A_{l m}$ reduces to $l(l+1)-s(s+1)$ in the Schwarzschild limit. The general series solution of the angular equation with appropriate boundary conditions is given in Appendix B1.

Let us consider the radial equation (4.5). For brevity, in the rest of the paper we drop the dependence of the Teukolsky function $R_{l m}$ on the angular indices $(l, m)$. Following Leaver (see Appendix B for details) we introduce three different solutions of the homogeneous equation (4.5): $R_{r_{+}}, R_{\infty_{+}}, R_{\infty_{-}}$, with asymptotic behavior [13]

$$
\begin{gathered}
\lim _{r \rightarrow r_{+}} R_{r_{+}} \sim\left(r_{+}-r_{-}\right)^{-1-s+i \omega+i \sigma_{+}} e^{i \omega r_{+}}\left(r-r_{+}\right)^{-s-i \sigma_{+}}, \\
\lim _{r \rightarrow \infty} R_{r_{+}} \sim A_{\mathrm{in}}^{T}(\omega) r^{-1-i \omega} e^{-i \omega r}+A_{\mathrm{out}}^{T}(\omega) r^{-1-2 s+i \omega} e^{i \omega r}, \\
\lim _{r \rightarrow \infty} R_{\infty_{+}} \sim r^{-1-2 s+i \omega} e^{i \omega r}, \\
\lim _{r \rightarrow \infty} R_{\infty_{-}} \sim r^{-1-i \omega} e^{-i \omega r} .
\end{gathered}
$$

In these relations $\sigma_{+}=\left(\omega r_{+}-a m\right) / b$ and $b=\sqrt{1-4 a^{2}}$. 
By definition, QNM frequencies $\omega_{n}$ are such that $A_{\mathrm{in}}^{T}\left(\omega_{n}\right)=0$. We compute them following the procedure in 11, 33. and verifying that $A_{\mathrm{in}}^{T}=0$ to some required numerical accuracy (typically better than one part in $10^{6}$ ). From (4.7b) and (4.8b) it follows that

$$
\begin{aligned}
& R_{r_{+}}=A_{\mathrm{in}}^{T} R_{\infty_{-}}+A_{\text {out }}^{T} R_{\infty_{+}}, \\
& R_{r_{+}}^{\prime}=A_{\mathrm{in}}^{T} R_{\infty_{-}}^{\prime}+A_{\text {out }}^{T} R_{\infty_{+}}^{\prime} .
\end{aligned}
$$

From these relations it is clear that computing the QNEFs requires accurate representations of the solutions both close to the horizon and at infinity. Close to the horizon, an accurate representation is provided by a series solution first found by Jaffé. At infinity we must resort to a different expansion in terms of Coulomb wavefunctions. Properties of these representations which are important for the calculation of the three solutions $\left(R_{r_{+}}, R_{\infty_{+}}\right.$and $\left.R_{\infty_{-}}\right)$are given in Appendix B2 and B3 respectively. We performed a number of consistency checks on the solutions, some of which are described in Appendix B4, More details can be found in the original papers by Leaver [11, 12, 13]. Notice that the outgoing-wave amplitude $A_{\text {out }}$ is unambiguously defined once we fix the normalization according to Eq. (4.7). The corresponding normalization for the SN wavefunctions is derived in Appendix Cl see in particular Eqs. (C7), (C13) and (16).

To compute the QNEFs (4.2) as functions of the rotation rate $j=2 a$, we first obtain the amplitudes $A_{\text {in }}^{T}$ and $A_{\text {out }}^{T}$ by matching the three solutions and their derivatives at some point (usually $r=5-7$ ). Then we compute the derivative $\alpha_{n}$ introduced in (3.8). Close to a QNM frequency we can perform a Taylor expansion of the ingoing amplitude: $A_{\mathrm{in}}^{T} \simeq \alpha_{n} \delta \omega$, with $\delta \omega$ a small complex number. For each value of $s$ and $a$ we evaluate $A_{\mathrm{in}}^{T}$ at a discrete set of points $\omega=\omega_{n}+k \delta \omega$ (typically we pick $\delta \omega=10^{-4}$ and the integer $k=-2, \ldots, 2$ ). Then we obtain $\alpha_{n}$ from a linear fit of $A_{\mathrm{in}}^{T}$ as a function of $\delta \omega$, and verify that the resulting derivatives satisfy the Cauchy-Riemann conditions.

In the rest of this paper we present our results for the QNEFs using both the Teukolsky and SN formalisms, and discuss some of their implications for gravitational-wave phenomenology.

\section{B. Numerical results}

The QNEFs of Schwarzschild black holes were first introduced and computed by Leaver in his seminal analysis of the radiative Green's function. Table I of [13] lists the QNEFs for the first four overtones of electromagnetic perturbations with $l=1$, the first seven overtones of gravitational perturbations with $l=2$ and the first four overtones of gravitational perturbations with $l=3$ and $l=4$. Building on Leaver's analysis, Sun and Price [14] studied the initial data dependence of the Schwarzschild quasinormal excitation coefficients. Andersson [17] computed the QNEFs for scalar and gravitational perturbations using the approximate phase-integral method, finding good quantitative agreement with Leaver for $s=-2$ and pointing out a few sign errors in Leaver's results. Later, using the same technique he was able to compute the (scalar) QNEFs up to overtone numbers $n \sim 200$ [18]. Glampedakis and Andersson [20] tried to extend the methods of Ref. [17] to scalar perturbations of Kerr black holes. The QNEFs listed in their Table 3 do not reduce to the correct Schwarzschild limit [34], and the "effective amplitude" results in Figure 5 of their paper are only qualitatively correct.

We computed QNEFs using both Mathematica and a Fortran code. In the limit $a \rightarrow 0$ the Fortran code reproduces Table I of [13], once we correct for Leaver's sign mistakes, to all (five) significant digits. Given the complexity of the algorithm described in Appendix B it is hard to quantify our numerical error for $a \neq 0$, but results should be accurate to the same level. The numerical accuracy depends on many factors, including the number of terms included in the Coulomb wavefunction expansion (B39), the matching radius chosen to solve the linear system (4.9) and the value of $\delta \omega$ used to compute $\alpha_{n}$ by a linear fit. In general, the number of digits to which $A_{\text {in }}=0$ at the QNM frequency and the level to which the Cauchy-Riemann conditions are satisfied in the calculation of $\alpha_{n}$ are good accuracy indicators. We decided to list the QNEFs with a five-digit accuracy, but in some cases (especially for corotating modes with $l=m$ and large rotation rates) the number of significant digits may be smaller.

\section{Scalar perturbations}

In Tables \ and [I] we provide the QNM frequencies and damping times for scalar perturbations with $l=2$, all values of $m$ and selected values of the angular momentum $j=2 a$. In Table III we list the corresponding QNEFs, and in Table IV the outgoing-wave amplitudes $A_{\text {out }}$. In the Schwarzschild limit, our QNEFs can be compared with Andersson's [17, 18]. His result for the fundamental scalar mode with $l=2$ agrees with ours to a five-digit accuracy [35]. This agreement is quite impressive, given the approximate nature of the phase-integral method. For $l=0$ his result for the fundamental mode $\left(B_{0}^{(0)} \simeq 0.197-0.046 i\right)$ is within $\sim 7 \%(\sim 23 \%)$ of the real (imaginary) part of our 
result: $B_{0}^{(0)} \simeq 0.212-0.059 i$. The agreement is still very good, considering that the phase-integral method is based on a WKB-type approximation and should only be accurate in the "eikonal" (large $l$ ) limit.
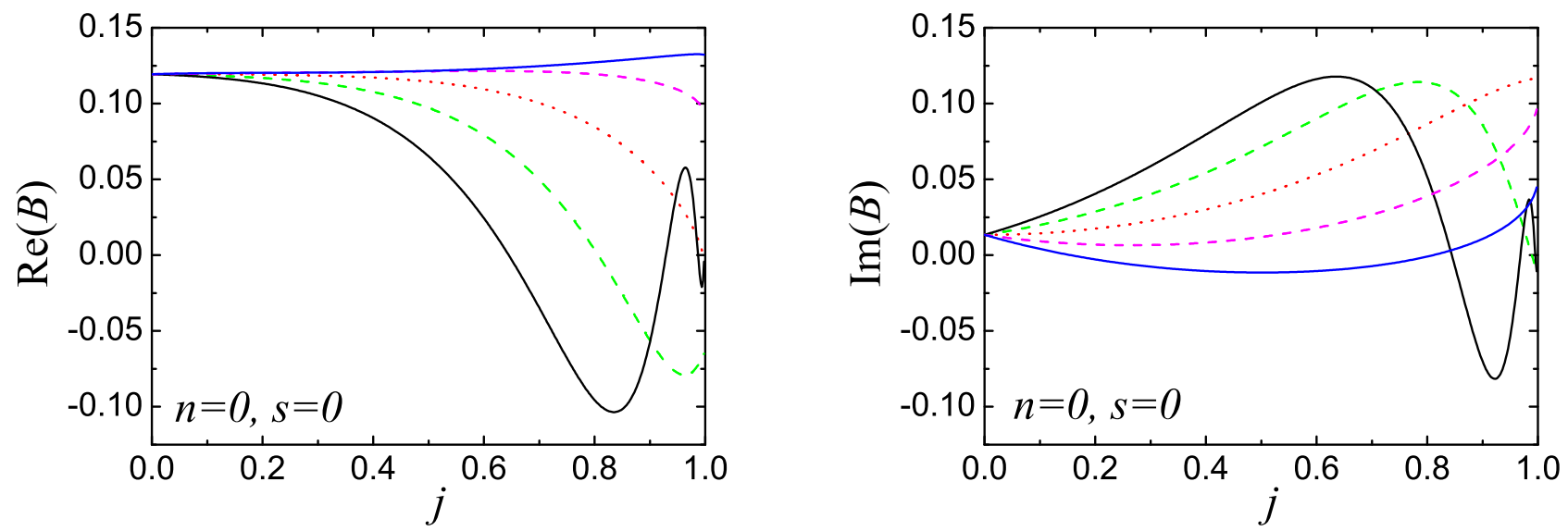

FIG. 3: Real part (left) and imaginary part (right) of the scalar QNEFs for the fundamental mode with $l=2$. Solid lines correspond to $|m|=2$, dashed lines to $|m|=1$, the dotted line to $m=0$. The lines displaying oscillations for large $j$ (black and green in the colour version) have $m>0$.

Some physical insight can be obtained by plotting the real and imaginary parts of the scalar QNEFs for the fundamental mode with $l=2$ as functions of $j$ (Fig. 3). The excitation of modes with $m \leq 0$ is a slowly-varying function of the rotation rate. On the contrary, as $j \rightarrow 1$ the real and imaginary parts of $B^{(\overline{0})}$ for corotating modes become rapidly oscillating functions of $j$. Our calculation is in remarkable agreement with a classic result by Ferrari and Mashhoon [36]: when $l=m$ the QNM excitation tends to zero as $j \rightarrow 1$, and this is an indication (if not a proof, given the incompleteness of QNMs) that extremal Kerr black holes are not marginally unstable.
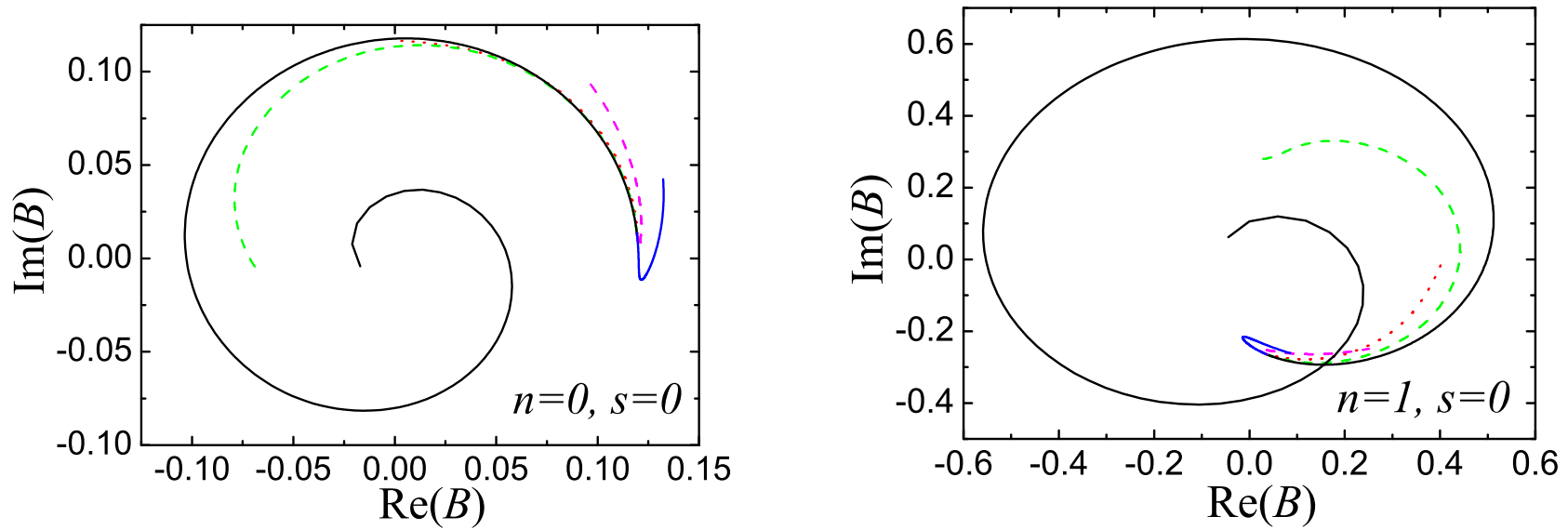

FIG. 4: Path of the scalar QNEFs for the fundamental mode (left) and first overtone (right) with $l=2$ as $j$ varies in the range $0 \leq j \leq 0.996$. Linestyles are the same as in Fig. 3

More features can be seen in Fig. 4, where we plot the path followed by the real and imaginary parts of $B_{n}^{(0)}$, thought of as parametric functions of $j$, for $0 \leq j \leq 0.996$. The oscillations of the real and imaginary parts of $B^{(0)}$ for the corotating mode with $l=m=2$ produce a spiral in the complex plane. For small $j$, the QNEFs of corotating 
and counterrotating modes with the same $|m|$ move in opposite directions, approaching the Schwarzschild limit with the same tangent. Corotating and counterrotating QNM frequencies tend to their Schwarzschild limit in a similar way [33, 37]. Another noteworthy feature of Fig. 4 is the scale of the real and imaginary axes: for corotating modes with $l=m$ and large rotation, $\left|B_{1}^{(0)}\right|$ is roughly five times larger than $\left|B_{0}^{(0)}\right|$. In other words, for perturbations with $l=m$ the contribution of the overtones should be more significant for rapidly rotating black holes. In the following we will see that many of these considerations are still valid when we consider perturbations of spin $s \neq 0$.

\section{Electromagnetic perturbations}

It is widely believed that astrophysical black holes should possess very little charge (if any). For this reason electromagnetic perturbations of a Kerr black hole $(s=-1)$ are not considered of great astrophysical relevance. Nonetheless, electromagnetic perturbations are of more than academic interest. For example, they could find useful applications in models of elementary particles based on the Kerr-Newman metric (see [38] and references therein).
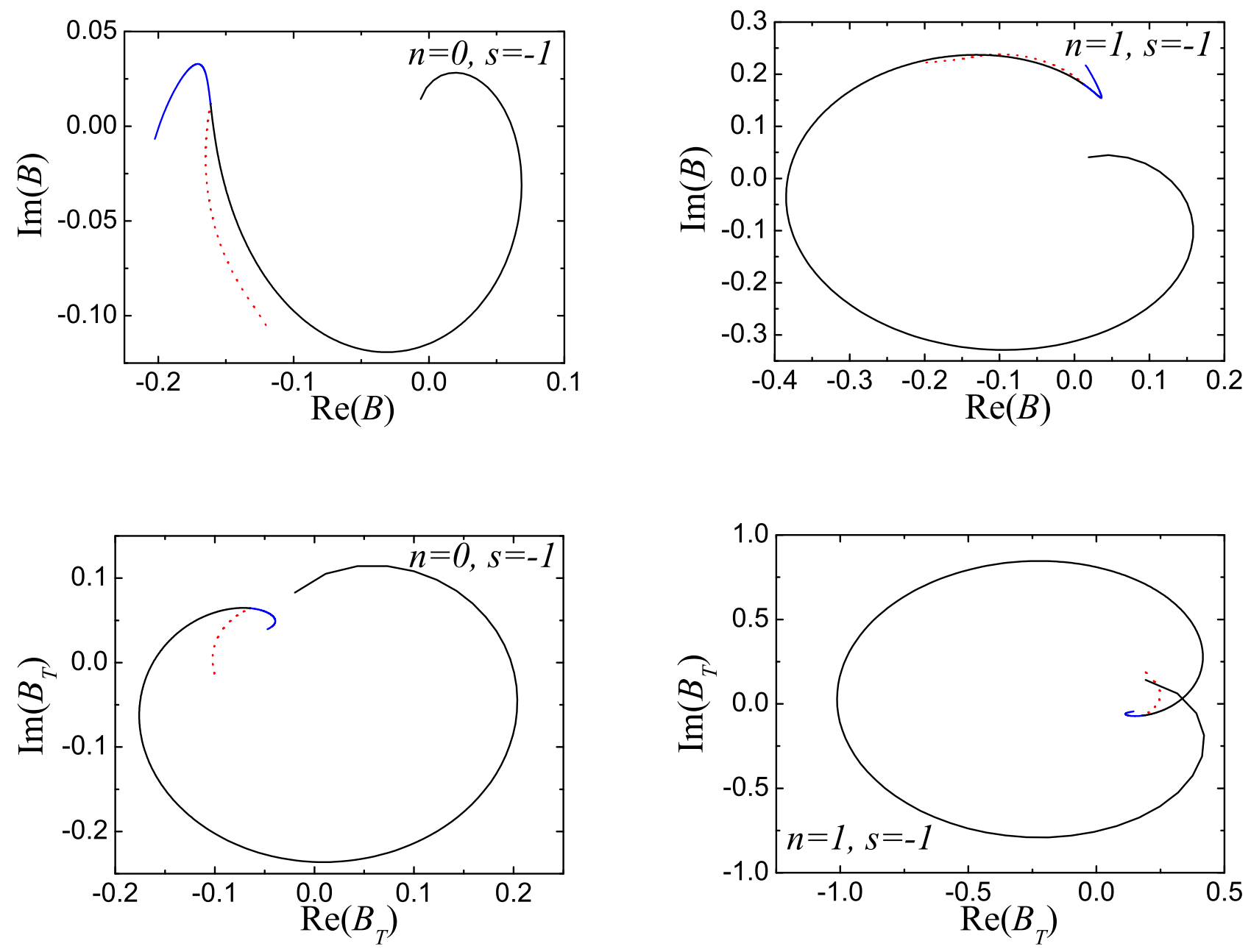

FIG. 5: Path of the electromagnetic QNEFs for the fundamental mode (left) and first overtone (right) with $l=1$ as $j$ varies in the range $0 \leq j \leq 0.996$. The top panels refer to the SN formalism, the bottom panels to the Teukolsky formalism. Linestyles are the same as in Fig. 3 .

Results for the lowest radiative multipole $l=1$ are presented in Fig. 5 . Compared with the scalar case, the main new feature is that QNEFs differ (both analytically and numerically) depending on whether we use the SN or 
Teukolsky formalisms: see Eq. (4.2) and Appendix C] Of course, both formalisms are equally legitimate, and the use of one or the other depends on the physical problem at hand. By construction the SN QNEFs reduce to the Regge-Wheeler QNEFs in the Schwarzschild limit, and in this sense they have a more direct physical interpretation. As $j \rightarrow 0$ the (Bardeen-Press-)Teukolsky quantities can be transformed to the corresponding metric quantities in the Zerilli (Regge-Wheeler) formalism using the differential transformations derived by Chandrasekhar [39].

Apart from this distinction, the qualitative features of Fig. 5 are similar to the scalar case of Fig. 4, For small $j$, the QNEFs of corotating and counterrotating modes with the same $|m|$ approach the Schwarzschild limit with the same tangent. For corotating modes with $l=m=1$ and large rotation rates, $\left|B_{1}^{(-1)}\right|$ is significantly larger than $\left|B_{0}^{(-1)}\right|$. This is another indication that, independently of the the value of $s$, the high-overtone contribution should be more significant for $l=m$ modes and rapidly rotating black holes.

\section{Gravitational perturbations}

Gravitational QNEFs in the Teukolsky and SN formalisms are listed in Tables V]VI] for the first two overtones with $l=2$, and selected values of the black hole's angular momentum. The corresponding QNM frequencies and angular separation constants can be found in Tables II and V of [4], respectively (there we list the imaginary parts with the opposite sign). As $j \rightarrow 0$ our numbers agree to all digits with Table I of [13], except for the relative sign of a few modes with $l=3$ and $l=4$. These minor sign errors in Leaver's paper were first pointed out in [17].

In Fig. 66 we plot the real and imaginary parts of the gravitational QNEFs for the first two overtones with $l=2$ in the SN formalism as functions of $j$. The qualitative behavior is remarkably similar to the scalar case (cf. Fig. 3). For fast rotation, the maximum excitation of the first gravitational overtone (as compared to the maximum excitation of the fundamental mode) is larger by a factor $\sim 8$.

In Fig. 7 we plot the path followed by the real and imaginary parts of $B_{n}^{(-2)}$, thought of as parametric functions of $j$, in the range $0 \leq j \leq 0.996$. There's a remarkable similarity between the plot for $n=0$ in the SN formalism and the scalar case of Fig. 4 . Even though it's hard to accurately compute the QNEFs when $j$ is very close to one, our numerics show clear evidence that in both cases the excitation of $l=m$ modes is zero in this limit: the center of the spiral is located at the origin of the complex plane. Our calculation provides further evidence that extremal Kerr black holes are stable, and that QNMs of a fast rotating Kerr black hole are very hard to excite [19, 20, 36].

In Fig. 8 we plot the modulus of gravitational and scalar QNEFs for the fundamental mode and first overtone with $l=m=2$. This plot is useful to visualize the effect of rotation on the excitation of different overtones, at least for radially localized initial data (for gaussian initial data the response is exponentially modulated by the QNM frequencies, see below). For gravitational perturbations the first overtone is maximally excited (with a relative amplification of a factor $\sim 8$ with respect to the fundamental mode) for $j \simeq 0.95$. For scalar perturbations the effect is similar, but the relative amplification of the first overtone is smaller (roughly a factor 6 ) and the maximum occurs at $j \simeq 0.85$.

\section{A simple application: localized and gaussian initial data}

As an application of our calculation of the QNEFs, we study the response of a Kerr black hole to initial data in simple situations amenable to an analytic treatment. The relevance of these model problems to realistic perturbations of astrophysical black holes is questionable, but our analysis could provide some insight into the black hole's response to more generic initial data. For simplicity in the following discussion we focus on scalar perturbations, so that Eq. (3.10) applies. We start by considering static, localized initial data of the form

$$
X^{(0)}\left(t_{0}, r\right)=\delta\left(r_{*}-r_{*}^{S}\right), \quad \dot{X}^{(0)}\left(t_{0}, r\right)=0 .
$$

Combining Eq. (3.7) with the definition (3.16) yields

$$
X^{(0)}(t, r)=-\operatorname{Re}\left[\left.\sum_{n=0}^{\infty} i B_{n} e^{-i \omega_{n}\left(t-t_{\text {start }}\right)} \frac{\hat{X}_{r_{+}}^{(0)}\left(r_{*}^{S}\right) e^{-i \omega_{n}\left|r_{*}^{S}\right|}}{A_{\text {out }}}\left(\omega_{n}-\frac{2 a m r+\omega_{n} a^{2} \gamma_{l m} \Delta}{\left(r^{2}+a^{2}\right)^{2}}\right)\right|_{r=r^{S}}\right] .
$$

For initial data in the far zone this is further simplified to

$$
X^{(0)}(t, r) \simeq-\operatorname{Re}\left[\sum_{n=0}^{\infty} i \omega_{n} B_{n} e^{-i \omega_{n}\left(t-t_{\text {start }}\right)}\right], \quad r_{*}^{S} \rightarrow+\infty .
$$



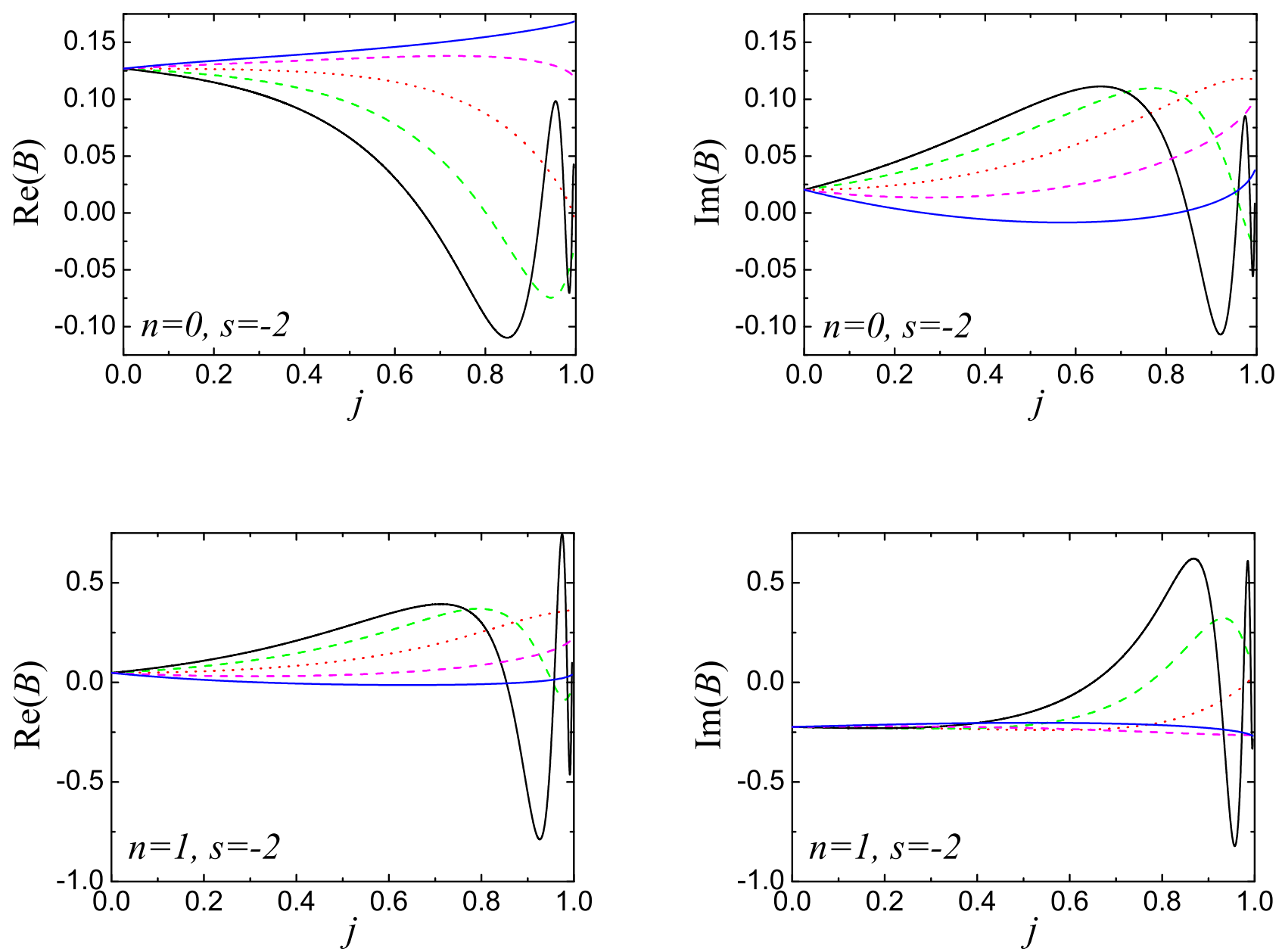

FIG. 6: Real part (left) and imaginary part (right) of the gravitational QNEFs with $l=2$ and different values of the overtone index $n$, indicated in the inset. All plots refer to the SN formalism. Linestyles are the same as in Fig. 3

For initial data localized near the horizon, using (3.3) we get

$$
X^{(0)}(t, r) \simeq-\operatorname{Re}\left[\sum_{n=0}^{\infty} i\left(\omega_{n}-2 m \Omega\right) \frac{B_{n}}{A_{\text {out }}} e^{-i \omega_{n}\left(t-t_{\text {start }}\right)} e^{i m \Omega r_{*}^{S}}\right], \quad r_{*}^{S} \rightarrow-\infty .
$$

Rotational effects appear (in the form of the usual Lense-Thirring frame dragging) only when the $\delta$-like disturbance is located near the horizon. Eq. (3.17) shows that in the Schwarzschild case $B_{n} \sim 1 / n$ for large $n$. Since we expect the same leading-order behavior to hold for Kerr black holes, and we know that Kerr QNM frequencies scale as $\omega_{n} \sim-i n$ for large $n$ [33], a $\delta$-like source excites all modes to comparable amplitude. This is reminiscent of the analogous result (2.201) for the vibrating string.

The vibrating string analogy suggests that we may be able to associate nodes to the QNM eigenfunctions. In other words, there could be special locations of the initial data $r_{*}^{S}$ for which the black hole's response is zero (or at least very small). We expect it should be easier to find these "nodes", if they exist at all, for QNM eigenfunctions which are as close as possible to ordinary normal mode eigenfunctions. This happens when $\operatorname{Re}\left(\omega_{n}\right) \gg \operatorname{Im}\left(\omega_{n}\right)$, that is, for corotating modes with $l=m$ and near-extremal $(j \simeq 1)$ black holes [37]. For this reason below we focus on scalar perturbations of Kerr black holes with $j=0.98$ and consider only modes with $l=m=1$ or $l=m=2$.

In Fig. 9 we plot the response of a Kerr black hole with $j=0.98$ (so that the horizon radius $r_{+} \sim 0.5994$ ) to initial data localized at different values of $r^{S}$, using Eq. (4.11). We compute the QNM frequency $\omega_{n}$ and the angular 

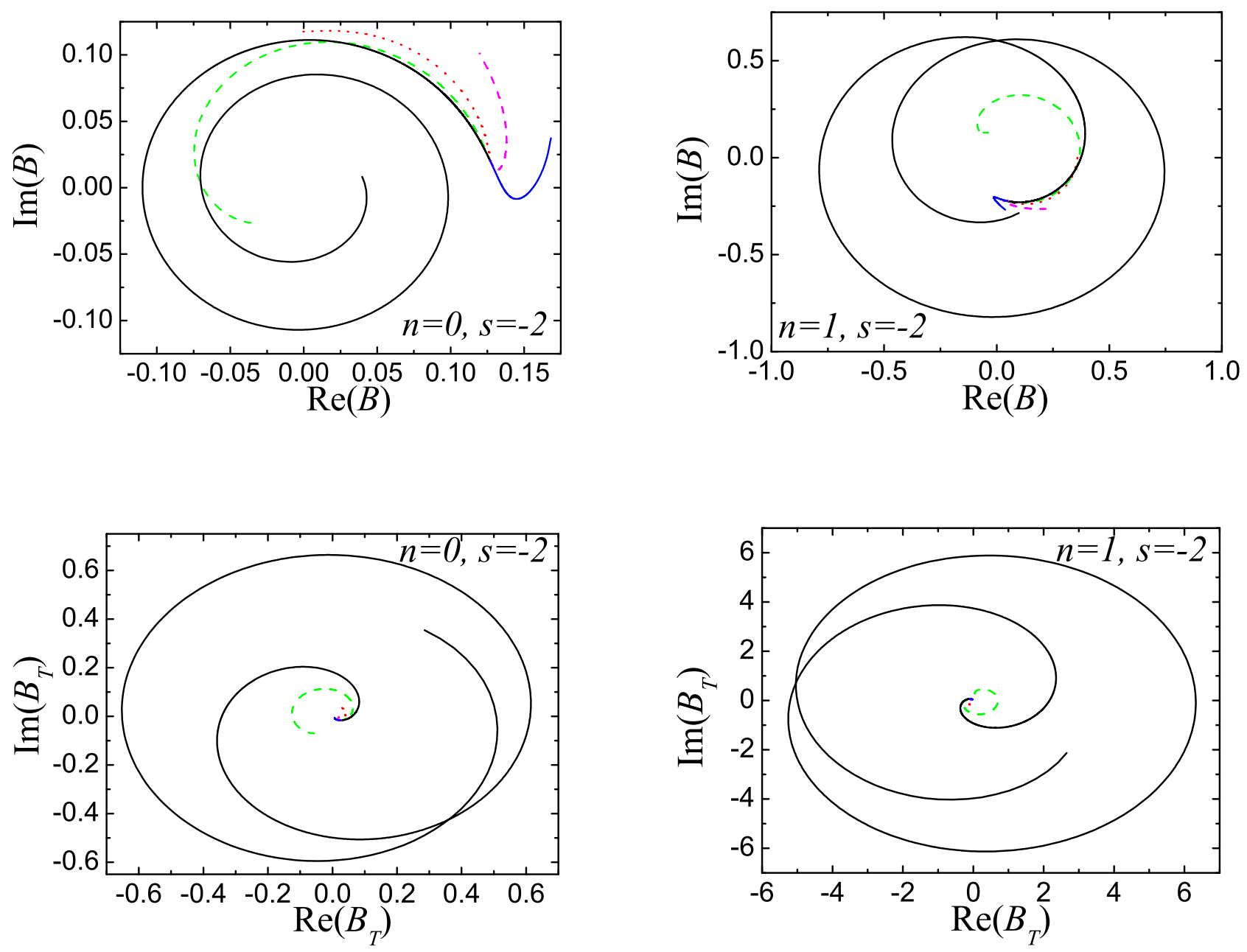

FIG. 7: Path of the gravitational QNEFs for the fundamental mode (left) and first overtone (right) with $l=2$ as $j$ varies in the range $0 \leq j \leq 0.996$. The top panels refer to the SN formalism, the bottom panels to the Teukolsky formalism. Linestyles are the same as in Fig. 3 .

eigenvalue $A_{l m}$ in (4.11) using Leaver's method [1]. The eigenfunction $\hat{X}_{r_{+}}^{(0)}\left(r_{*}^{S}\right)$ can be obtained combining Eq. (B8) and (C3). The values of $B_{n}$ and $A_{\text {out }}$ can be read off Tables [II and IV] respectively. For increased accuracy we evaluate the $\gamma_{l m}$ 's numerically from their definition (3.11), integrating the (complex) angular QNM eigenfunctions (see Appendix B 1), but the numerical results differ only marginally from the simple analytic approximation (3.12).

For fixed $(l, m)$ and $n=0$ the black hole's response has a relative maximum when the initial data are located very close to the horizon. As $r^{S}$ increases the wave amplitude decreases monotonically, reaching a minimum at $r^{S} \simeq 0.86$ (the exact location is only weakly sensitive to the particular mode we consider). This radial location can be seen as corresponding to a "node" of the QNM eigenfunction. We also show wave amplitudes for initial data located relatively far from the horizon, at $r^{S}=2$. These amplitudes are already quite close to the amplitudes for initial data "falling from infinity".

The association of nodes with the eigenfunctions is more problematic and ambiguous for larger damping. For example, if we consider the fundamental mode of a Schwarzschild black hole (for which $\operatorname{Re}\left(\omega_{n}\right)$ and $\operatorname{Im}\left(\omega_{n}\right)$ are comparable) the wave amplitude turns out to be only weakly sensitive to the location $r^{S}$ of initial data. The same happens when we consider counterrotating modes of (slowly or rapidly rotating) Kerr black holes.

A natural next step is to study non-localized initial data. We consider static initial data given by a gaussian 


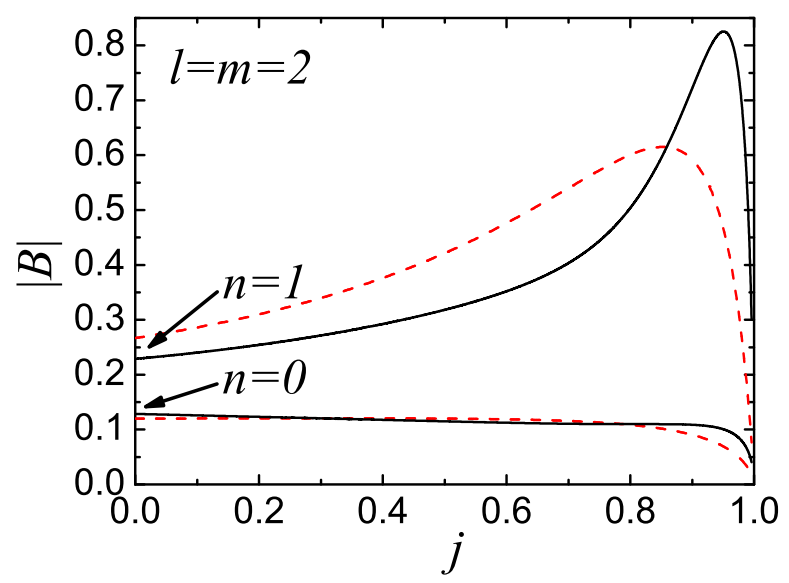

FIG. 8: Modulus of the SN QNEFs for the fundamental mode and first overtone with $l=m=2$. Solid (black) lines are for gravitational perturbations, dashed (red) lines for scalar perturbations.
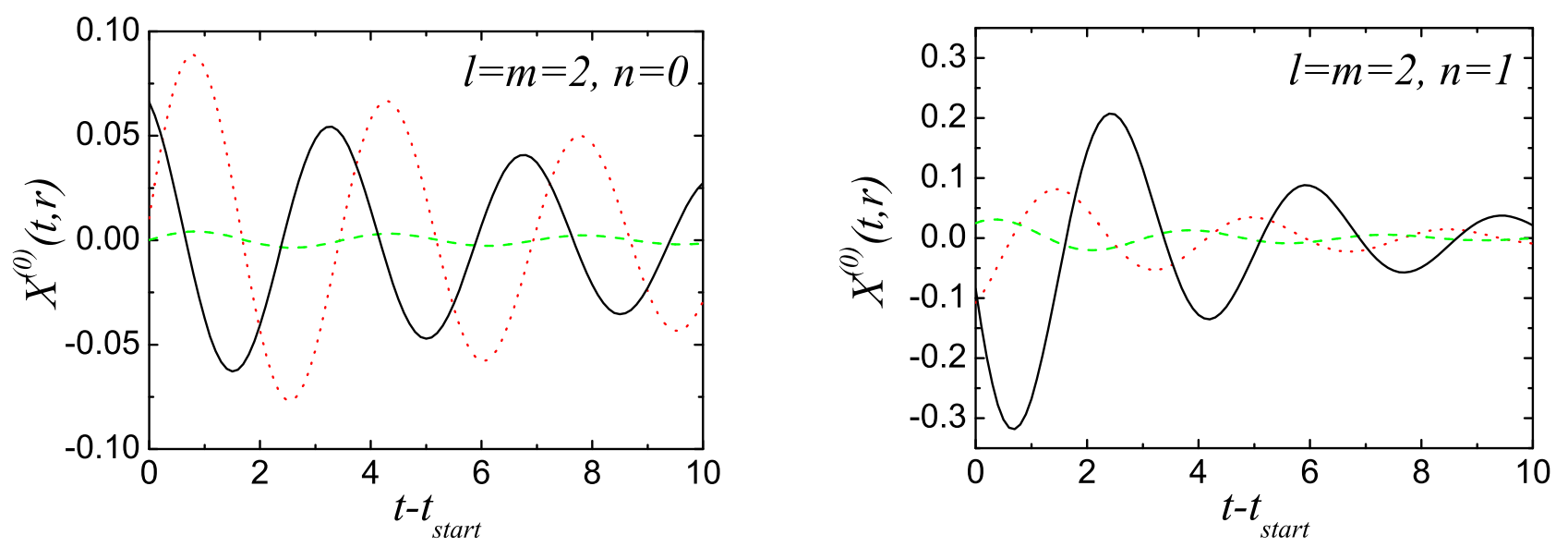

FIG. 9: Time-domain single-mode waveforms for scalar perturbations of a Kerr black hole with $j=0.98$. Each panel shows results for different values of $(l, m, n)$. Dotted (red) lines correspond to initial data located extremely close to the horizon, at $r^{S}=0.6$. Solid (black) lines are obtained for initial data localized relatively far away from the black hole, at $r^{S}=2$. Dashed (green) lines show the response for initial data localized at the point of minimal excitation, that we determined to be: $r^{S} \simeq 0.86$ $(l=m=2, n=0) ; r^{S} \simeq 0.87(l=m=2, n=1)$.

wavepacket

$$
X^{(0)}\left(t_{0}, r\right)=k e^{-b\left(r_{*}-r_{*}^{S}\right)^{2}}
$$

where $k$ and $b$ are arbitrary constants. For Schwarzschild black holes, the scattering of gaussian initial data was first studied numerically in the time domain by Vishveshwara [10]. An interpretation of the results in terms of QNM expansions for initial data in the far zone was later provided by Andersson [17]. Following [17], for initial data in the 
far zone (large $r_{*}^{S}$ ) we get

$$
\begin{aligned}
X^{(0)}(t, r) & \simeq-\operatorname{Re}\left[\sum_{n=0}^{\infty} i \omega_{n} B_{n} e^{-i \omega_{n}\left(t-t_{\mathrm{start}}\right)} e^{-i \omega_{n} r_{*}^{S}} \int_{-\infty}^{+\infty} e^{i \omega_{n} r_{*}} k e^{-b\left(r_{*}-r_{*}^{S}\right)^{2}} d r_{*}\right] \\
& =-\operatorname{Re}\left[\sum_{n=0}^{\infty} i \omega_{n} k B_{n} \sqrt{\frac{\pi}{b}} e^{-\frac{\omega_{n}^{2}}{4 b}} e^{-i \omega_{n}\left(t-t_{\mathrm{start}}\right)}\right] .
\end{aligned}
$$

If the gaussian is centered very close to the black hole $\left(r_{*}^{S} \ll 0\right)$ we have

$$
X^{(0)}(t, r) \simeq-\operatorname{Re}\left[\sum_{n=0}^{\infty} \frac{i\left(\omega_{n}-2 m \Omega\right) k B_{n}}{A_{\text {out }}} e^{i m \Omega r_{*}^{S}} \sqrt{\frac{\pi}{b}} e^{-\frac{\left(\omega_{n}-m \Omega\right)^{2}}{4 b}} e^{-i \omega_{n}\left(t-t_{\text {start }}\right)}\right] .
$$

Once again, frame dragging effects show up only for gaussians located near the horizon. The absolute value of each term in the sum is proportional to $\exp [-\operatorname{Re}(w) / 4 b]$, with $w=\omega_{n}^{2}$ or $w=\left(\omega_{n}-m \Omega\right)^{2}$ depending on the location of the initial data. Minimizing with respect to $b$ we see that the maximal excitation corresponds to $b=\operatorname{Re}(w) / 2$ : we found a similar result in the vibrating string example [recall the discussion below Eq. (2.22)].

In the limit of narrow gaussians we expect to recover the results for localized initial data. Indeed, if we use the $\delta$-function representation setting $k=(2 \sqrt{\pi \epsilon})^{-1}$ and $b=1 /(4 \epsilon)$ :

$$
\delta\left(r_{*}-r_{*}^{S}\right)=\frac{1}{2 \sqrt{\pi \epsilon}} e^{-\frac{\left(r_{*}-r_{*}^{S}\right)^{2}}{4 \epsilon}},
$$

by taking the limit $\epsilon \rightarrow 0$ we recover (4.12) and (4.13).

\section{Effective amplitude and initial data}

The present calculation of the QNEFs is only a first step towards the determination of the QNM content of a waveform. The degree to which a given QNM is excited depends crucially, through the integral appearing in (3.9), on the initial data $I(\omega, r)$. Can we identify some physical quantity which is only weakly dependent on the initial data, and therefore useful to discuss the detectability of QNMs?

Andersson and Glampedakis proposed to use a QNM "effective amplitude" defined as follows [19, 20]. Consider a typical ringdown waveform $\Psi$. Matched filtering increases the gravitational wave amplitude by $\sqrt{N}$, $N \propto \operatorname{Re}\left(\omega_{n}\right) / \operatorname{Im}\left(\omega_{n}\right)$ being the number of cycles, yielding an effective amplitude $\Psi_{\text {eff }} \sim \sqrt{N} \Psi$. For a single mode $\Psi \propto C_{n}$, so that $\Psi_{\text {eff }} \sim \sqrt{N} C_{n}$. Now, if we happen to know that the convolution of the initial data with the homogeneous solution is reasonably independent of $\omega_{n}$, we could write

$$
\Psi_{\text {eff }} \sim \sqrt{\operatorname{Re}\left(\omega_{n}\right) / \operatorname{Im}\left(\omega_{n}\right)} B_{n},
$$

which would be the sought criterion for detectability of a given mode. In [19, 20] the real part of (4.18) was referred to as the "effective gravitational wave amplitude", and used to discuss QNM detectability.

The derivation of Eq. (4.18) relies upon the assumption that the proportionality factor between the $C_{n}$ 's and the $B_{n}$ 's is weakly dependent on $\omega_{n}$, for only in this case is the previous expression valid. Eqs. (4.12) and (4.13) show that such an assumption is reasonable for localized initial data. Unfortunately this won't be true in more general situations. A simple counterexample is provided by gaussian initial data. According to (4.18) QNM detectability should be weakly dependent on $\omega_{n}$ (at least for low-lying modes), but we know for a fact that the correct answer for gaussian initial data, Eq. (4.15), has an exponential dependence on $\omega_{n}$. Any notion of detectability based on the naive argument leading to (4.18) is therefore dangerous, and a case-by-case analysis of the initial data is mandatory. A study of the black hole's response to physically reasonable initial data will be the subject of future work.

\section{CONCLUSIONS AND OUTLOOK}

Motivated by the prospect to test the Kerr nature of astrophysical black holes by gravitational wave observations, in this paper we compute QNEFs for general-spin perturbations of Kerr black holes. For corotating modes with $l=m$ we find that QNEFs tend to zero in the extremal limit, and that the overtone contribution is more significant when the black hole is fast rotating. This result is confirmed by numerical time-evolutions of scalar perturbations of Kerr 
black holes [40]. In Appendix $\mathrm{A}$ we also present the first analytical calculation of the large- $n$ QNEFs for static black holes, including the Schwarzschild and Reissner-Nordström metrics.

QNEFs are "universal" properties of the Kerr metric: they do not depend on the physical nature of the perturbation. Suppose that a distorted Kerr black hole is formed as a result of a binary merger. The detailed physics of the merger, depending on parameters such as the masses, spin magnitude and inclination of the binary members, will affect the relative overtone excitation through the initial data function $I(\omega, r)$ appearing in the definition (3.9) of the excitation coefficients. Therefore the problem of determining the relative overtone excitation in ringdown waveforms reduces to the determination of the function $I(\omega, r)$ from numerical simulations.

Of course the present formalims relies on the possibility to use linear perturbation theory to extract ringdown waveforms from numerical simulations. In the nonlinear regime the total mass and angular momentum of the dynamical spacetime could deviate significantly from their values for the final Kerr black hole, introducing systematic (and perhaps time-dependent) redshifts in the QNM spectrum [41]. An invariant criterion to monitor the applicability of linear perturbation theory is to use the Petrov classification, looking at the relative deviation of the background spacetime from Type D. The Petrov type of the background can be determined, for example, using a wave-extraction formalism based on the quasi-Kinnersley tetrad and looking at deviations of the scalar invariant $S$ from the value $(S=1)$ that it would have for a Kerr black hole [42].

A significant technical challenge in the calculation of the excitation coefficients $C_{n}$ is the renormalization of the divergent integral (3.9) for generic initial data. We will address this problem in future publications. As a testbed we plan to study simple models amenable to perturbation theory (eg. the old problem of particles plunging into Kerr black holes). Then we wish to explore the nonlinear regime in relatively symmetric situations, such as head-on collisions or nonlinear simulations of single, distorted black holes. Finally we will consider the harder, astrophysically realistic problem of determining the relative overtone excitation from nonlinear simulations of merging black holes, using Newman-Penrose based wave extraction of the ringdown waveform from full numerical relativity simulations.

An accurate determination of the relative QNM excitation in realistic binary mergers would have interesting astrophysical applications. For example it could be used to assess the feasibility of tests of the no-hair theorem with Earth-based and space-based gravitational wave interferometers, and to improve present estimates of the "ringdown braking" phase observed in numerical and analytical calculations of gravitational wave recoil [43, 44].

\section{Acknowledgements}

We thank Amanda Gonzales and Ana Sousa for useful discussions. We are grateful to Nils Andersson, Kostas Glampedakis, Cliff Will, the relativity group at Louisiana State University and the participants of the workshop on Astrophysical Applications of Numerical Relativity in Guanajuato (Mexico) for their comments and constructive criticism. VC acknowledges financial support from the Fundação Calouste Gulbenkian through Programa Gulbenkian de Estímulo à Investigação Científica. This work was supported in part by the National Science Foundation under grant PHY 03-53180, and by NASA grant NNG06GI60.

\section{APPENDIX A: ASYMPTOTIC QUASINORMAL EXCITATION FACTORS OF STATIC BLACK HOLES}

One of the fundamental prerequisites in order to have a well-defined QNM expansion is that the QNEFs $B_{n}$ "converge" in some sense. So far this problem has not received much attention. A numerical study of the scalar QNEFs for large $n$ and non-rotating black holes can be found in [18]. In this Appendix we study analytically the large- $n$ behavior of the QNEFs for Schwarzschild black holes and other static geometries. We explicitly compute the dependence of the QNEFs on $n$ (including subleading effects) in three important cases, including the Schwarzschild and Reissner-Nordström spacetimes and the widely used Pöschl-Teller approximation of the Regge-Wheeler potential. We also show that for a general class of static spacetimes the leading behavior of the QNEFs for large $n$ is $\sim 1 / n$, and we conjecture that the same asymptotic behavior should apply to Kerr black holes. It should be easy to generalize our technique to compute subleading effects. Finally, as a simple counterexample to the generality of this behavior, we show that the QNEFs for a potential barrier scale as $1 / n^{3}$ for large $n$.

\section{Schwarzschild black holes}

Our general method relies on the monodromy argument by Motl and Neitzke [45], devised to compute highly damped (i.e. large- $n$ ) QNM frequencies (see [37] and [46] for reviews). Using this method Neitzke computed the 
reflection and transmission coefficients $R$ and $T$ for large $n$ [47]. From his calculation we can simply read off the in-going and out-going wave amplitudes for the Regge-Wheeler equation:

$$
\begin{aligned}
A_{\text {in }} & =\frac{1}{T}=\frac{e^{\beta \omega}+1+2 \cos (\pi s)}{e^{\beta \omega}-1}, \\
A_{\text {out }} & =\frac{R}{T}=\frac{2 i \cos (\pi s / 2)}{e^{\beta \omega}-1}
\end{aligned}
$$

where in our units $(G=c=2 M=1) \beta=4 \pi$ is the inverse of the black hole's Hawking temperature. This result is valid for large $n$ and for any angular quantum number $l$. QNM frequencies, being poles of the reflection and transmission coefficients, satisfy

$$
e^{\beta \omega_{n}}+1+2 \cos (\pi s)=0
$$

The calculation of the QNEFs $B_{n}$ for large $n$ is now straightforward. Denoting the large- $n$ limit of the QNEFs for spin- $s$ perturbations by $B_{n}^{(s)}$ we find

$$
B_{n}^{(s)} \equiv\left(\frac{A_{\text {out }}}{2 \omega \partial_{\omega} A_{\text {in }}}\right)_{\omega=\omega_{n}}=\frac{i \cos (\pi s / 2)}{\beta \omega_{n} e^{\beta \omega_{n}}} .
$$

This leads to the result anticipated in the main text, Eq. (3.17):

$$
B_{n}^{(0)}=B_{n}^{(-2)}=-\frac{i}{3[ \pm \log 3-(2 n+1) \pi i]}, \quad(n \rightarrow \infty) .
$$

This is our main result for the Schwarzschild QNEFs in the large damping limit. The analytic prediction agrees very well with Figure 2 in [18], where the QNEFs are plotted for the first $\sim 200$ scalar modes. The calculation of Ref. [18] shows that for large $n$ QNEFs are independent of $l$ and drop linearly with $n$, in agreement with our prediction. Notice also that for electromagnetic perturbations the asymptotic QNEFs $B_{n}^{(-1)}$ would be zero: higher-order corrections must be taken into account to obtain a nontrivial result.

\section{Reissner-Nordström black holes}

The monodromy technique is easily adapted to other black hole backgrounds [48], allowing the computation of the asymptotic QNEFs for a wide class of geometries. Take for instance the Reissner-Nordström metric, describing a black hole with mass $M$ and charge $Q$. We follow Motl and Neitzke [45] and fix units such that $M=(k+1) / 2$ and $Q^{2}=k$. With this choice the outer horizon is located at $r=1$, and we get [47]

$$
\begin{aligned}
A_{\mathrm{in}} & =\frac{1}{T}=\frac{e^{\beta \omega}+2+3 e^{-\beta_{I} \omega}}{e^{\beta \omega}-1} \\
A_{\mathrm{out}} & =\frac{R}{T}= \pm i \sqrt{3} \frac{1+e^{-\beta_{I} \omega}}{e^{\beta \omega}-1}
\end{aligned}
$$

for any massless integer-spin field (at odds with the Schwarzschild case, where this symmetry is broken for electromagnetic perturbations). Here $\beta=4 \pi /(1-k)$ and $\beta_{I}=-k^{2} \beta$ denote the Hawking temperatures of the outer and inner horizons, respectively. For large $n$ the QNM frequencies $\omega_{n}$ are solutions of

$$
e^{\beta \omega}+2+3 e^{-\beta_{I} \omega}=0
$$

An elementary calculation similar to the Schwarzschild case yields

$$
B_{n}^{(s)}=\frac{ \pm i \sqrt{3}}{2 \omega_{n}} \frac{1+e^{-\beta_{I} \omega_{n}}}{\beta e^{\beta \omega_{n}}-3 \beta_{I} e^{-\beta_{I} \omega_{n}}}, \quad(n \rightarrow \infty) .
$$

In (A7) and (A9) the plus sign refers to electromagnetic-gravitational perturbations, and the minus sign refers to scalar perturbations. The fact that in the large- $n$ limit the predictions for charged black holes do not reduce to the Schwarzschild predictions as $Q \rightarrow 0$ is known, and agrees with numerical results [33]. In general, the transcendental equation (A8) must be solved numerically to obtain the QNM frequencies. As in the Schwarzschild case, one can verify that the QNEFs $B_{n} \sim 1 / n$. 


\section{The Pöschl-Teller potential}

The Pöschl-Teller potential has been used in many studies of black hole perturbation theory as a useful approximation of the Regge-Wheeler potential. The idea is to replace the "true" potential barrier by an analytic expression of the form $V=V^{\mathrm{PT}} \equiv V_{0} \cosh ^{-2} \alpha\left(x-x_{0}\right)$, which has the advantage that many calculations can be carried out in closed form [36, 49]. From the analytic expression of the reflection and transmission coefficients given in [36] we get

$$
\begin{aligned}
A_{\mathrm{out}} & =\frac{\Gamma(1+i \omega / \alpha) \Gamma(-i \omega / \alpha)}{\Gamma(1+\beta) \Gamma(-\beta)} \\
A_{\mathrm{in}} & =\frac{\Gamma(1+i \omega / \alpha) \Gamma(i \omega / \alpha)}{\Gamma(1+\beta+i \omega / \alpha) \Gamma(-\beta+i \omega / \alpha)}
\end{aligned}
$$

where $\beta=-1 / 2+\sqrt{1 / 4-V_{0} / \alpha^{2}}$. As usual, the QNM frequencies are poles of $A_{\text {in }}$. They can be obtained imposing (say) $\Gamma(-\beta+i \omega / \alpha)^{-1}=0$ (or equivalently $\left.-\beta+i \omega / \alpha=-n\right)$. Since $B_{n} \sim\left(\partial_{\omega} A_{\text {in }}\right)^{-1}$, the only nonzero contribution to $B_{n}$ must come from the term $[\Gamma(1+i \omega / \alpha) \Gamma(i \omega / \alpha) / \Gamma(1+\beta+i \omega / \alpha)] \partial_{\omega}(1 / \Gamma(-\beta+i \omega / \alpha))$. Introducing the polygamma function $\psi_{0}$, such that $\Gamma^{\prime}(x)=\Gamma(x) \psi_{0}(x)$, the derivative we need is

$$
\frac{d}{d \omega}\left[\frac{1}{\Gamma(-\beta+i \omega / \alpha)}\right]=-\frac{i \psi_{0}(-n)}{\alpha \Gamma(-n)}=\frac{(-1)^{n} i n !}{\alpha},
$$

and the final result for the asymptotic QNEFs of the Pöschl-Teller potential reads

$$
B_{n}^{\mathrm{PT}}=\frac{i \alpha(-1)^{n+1}}{2 \omega_{n} \Gamma(1+\beta) \Gamma(-\beta)} \frac{\Gamma(n-\beta) \Gamma(1+2 \beta-n)}{n ! \Gamma(\beta-n)} .
$$

Once again, asymptotically $B_{n}^{\mathrm{PT}} \sim 1 / n$.

\section{Generic static black hole spacetimes and other possible extensions}

An investigation of generic spacetimes with the present technique should be carried out case by case [48. However, if we are only interested in the leading-order behavior for large $n$ we can content ourselves with a simple Born approximation to the scattering amplitude (see for instance [50]) to deduce that $\omega \sim-$ in, so that generically the QNEFs $B_{n} \sim 1 / n$ for large $n$.

The generalization of the present results to Kerr black holes is more difficult. Previous numerical calculations of the asymptotic QNM frequencies [33, 37] show that the imaginary part is still proportional to $n$, so we expect that (to leading order) the Kerr QNEFs should still scale as $B_{n}^{\mathrm{Kerr}} \sim 1 / n$.

\section{A potential barrier}

As a last example we consider the QNEFs for a potential barrier of height $V_{0}$ and width $\bar{x}$, i.e. the wave equation is $\partial_{x}^{2} \Psi+\left[\omega^{2}-V(x)\right] \Psi=0$, with potential

$$
V(x)= \begin{cases}V_{0} & \text { for } 0<x<\bar{x} \\ 0 & \text { for } x<0 \text { and } x>\bar{x}\end{cases}
$$

Defining $k=\sqrt{\omega^{2}-V_{0}^{2}}$, the solution in each of the three different regions is

$$
\begin{aligned}
& \Psi=e^{-i \omega x}, \quad x<0, \\
& \Psi=A e^{i k x}+B e^{-i k x}, \quad 0<x<\bar{x} \\
& \Psi=C e^{i \omega x}+D e^{-i \omega x}, \quad 0<x<\bar{x} .
\end{aligned}
$$

The constants $A, B, C, D$ are obtained by imposing continuity of the field and its derivative at $x=0$ and $x=\bar{x}$, so that $A+B=1$ and $-\omega=k(A-B) /(A+B)$. QNM frequencies are such that $D(\omega)=0$. This problem was considered by Chandrasekhar and Detweiler [51], who showed that for large mode number $n$ the QNM frequencies behave as $\left(n \pi-i \omega_{i}\right)$, with $\omega_{i}$ a solution to the transcendental equation $\sqrt{V_{0}}=-2 \omega_{i} e^{\omega_{i} / 2}$. We have solved the differential 
equation numerically, and our results agree very well with this prediction. For large overtone $n$ we find the analytical result

$$
\begin{aligned}
& C \sim \frac{V_{0}}{4 k \omega} e^{-i \bar{x}(\omega+k)}\left(1-e^{2 i \bar{x} k}\right) \\
& D \sim-\frac{e^{i \bar{x}(\omega-k)}}{16 k \omega^{3}}\left(V_{0}^{2} e^{2 i \bar{x} \omega}-16 \omega^{4}\right) .
\end{aligned}
$$

We also get

$$
\frac{d D}{d \omega} \sim-\frac{e^{i \bar{x}(\omega-k)}}{16 k \omega^{3}}\left(2 i \bar{x} e^{2 i \bar{x} \omega} V_{0}^{2}-64 \omega^{3}\right)
$$

At the QNM frequencies $V_{0}^{2} e^{2 i \bar{x} \omega}-16 \omega^{4}=0$, and we get the following QNEFs

$$
B_{n}^{\text {barrier }}=\frac{C}{2 \omega \frac{d D}{d \omega}} \sim \frac{V_{0}}{32 i \bar{x} \omega^{3}}
$$

where we used the fact that in this regime $\operatorname{Im}(\omega) \rightarrow-\infty$ and $e^{-i \bar{x}(\omega+k)} \sim 0$. The result (A21) is in good agreement with numerical results and shows that the $1 / n$ behavior, although very general, is not universal, for in this case the QNEFs scale as $1 / n^{3}$.

\section{APPENDIX B: DETAILS ON THE CALCULATION OF THE QUASINORMAL EXCITATION FACTORS}

This Appendix provides details on the calculation of QNEFs for general-spin perturbations of Kerr black holes. Most of the material can be found in the original papers by Leaver [11, 12, 13] (see also [8, 33, 37]), but we find it convenient to summarize here the equations which are necessary to implement the computational procedure.

\section{Expansion of the angular wavefunction}

If we express the angular wavefunction $S_{l m}$ as

$$
S_{l m}(u)=e^{a \omega u}(1+u)^{\frac{|m-s|}{2}}(1-u)^{\frac{|m+s|}{2}} \sum_{n=0}^{\infty} a_{n}^{\theta}(1+u)^{n}
$$

the coefficients $a_{n}^{\theta}$ must satisfy the following recurrence relation

$$
\begin{aligned}
\alpha_{0}^{\theta} a_{1}^{\theta}+\beta_{0}^{\theta} a_{0}^{\theta} & =0, \\
\alpha_{n}^{\theta} a_{n+1}^{\theta}+\beta_{n}^{\theta} a_{n}^{\theta}+\gamma_{n}^{\theta} a_{n-1}^{\theta} & =0,
\end{aligned}
$$

where

$$
\begin{aligned}
\alpha_{n}^{\theta}= & -2(n+1)\left(n+2 k_{1}+1\right), \\
\beta_{n}^{\theta}= & n(n-1)+2 n\left(k_{1}+k_{2}+1-2 a \omega\right)- \\
& {\left[2 a \omega\left(2 k_{1}+s+1\right)-\left(k_{1}+k_{2}\right)\left(k_{1}+k_{2}+1\right)\right]-a^{2} \omega^{2}-s(s+1)-A_{l m}, } \\
\gamma_{n}^{\theta}= & 2 a \omega\left(n+k_{1}+k_{2}+s\right) .
\end{aligned}
$$

Regularity of the solution at the boundaries implies that the sequence of expansion coefficients must be minimal, and that the separation constant $A_{l m}$ must be a root of the continued fraction

$$
0=\beta_{0}^{\theta}-\frac{\alpha_{0}^{\theta} \gamma_{1}^{\theta}}{\beta_{1}^{\theta}-} \frac{\alpha_{1}^{\theta} \gamma_{2}^{\theta}}{\beta_{2}^{\theta}-} \frac{\alpha_{2}^{\theta} \gamma_{3}^{\theta}}{\beta_{3}^{\theta}-} \ldots
$$

or any of its inversions. 


\section{Jaffé expansion of the radial wavefunction}

As discussed below Eq. (4.9), to compute the Kerr QNEFs we need an accurate representation of the solutions of the radial equation. A convenient series solution close to the horizon can be found by methods due to Jaffé (see [1]]):

$$
R_{r_{+}}=e^{i \omega r}\left(r-r_{-}\right)^{-1-s+i \omega+i \sigma_{+}}\left(r-r_{+}\right)^{-s-i \sigma_{+}} \sum_{n=0}^{\infty} a_{n}^{r}\left(\frac{r-r_{+}}{r-r_{-}}\right)^{n}
$$

where the notation is the same as in Sec. IVA. The coefficients $a_{n}^{r}$ are normalized so that $a_{0}^{r}=1$, consistently with Eq. (4.7a). They can be obtained from the recurrence relation

$$
\begin{aligned}
\alpha_{0}^{r} a_{1}^{r}+\beta_{0}^{r} a_{0}^{r} & =0 \\
\alpha_{n}^{r} a_{n+1}^{r}+\beta_{n}^{r} a_{n}^{r}+\gamma_{n}^{r} a_{n-1}^{r} & =0,
\end{aligned}
$$

where

$$
\begin{aligned}
\alpha_{n}^{r} & =n^{2}+\left(c_{0}+1\right) n+c_{0}, \\
\beta_{n}^{r} & =-2 n^{2}+\left(c_{1}+2\right) n+c_{3}, \\
\gamma_{n}^{r} & =n^{2}+\left(c_{2}-3\right) n+c_{4}-c_{2}+2,
\end{aligned}
$$

and

$$
\begin{aligned}
& c_{0}=1-s-i \omega-\frac{2 i}{b}\left(\frac{\omega}{2}-a m\right), \\
& c_{1}=-4+2 i \omega(2+b)+\frac{4 i}{b}\left(\frac{\omega}{2}-a m\right), \\
& c_{2}=s+3-3 i \omega-\frac{2 i}{b}\left(\frac{\omega}{2}-a m\right), \\
& c_{3}=\omega^{2}\left(4+2 b-a^{2}\right)-2 a m \omega-s-1+(2+b) i \omega-A_{l m}+\frac{4 \omega+2 i}{b}\left(\frac{\omega}{2}-a m\right), \\
& c_{4}=s+1-2 \omega^{2}-(2 s+3) i \omega-\frac{4 \omega+2 i}{b}\left(\frac{\omega}{2}-a m\right) .
\end{aligned}
$$

QNM frequencies satisfy a continued-fraction relation analogous to (B7), with the superscript $\theta$ replaced by a superscript $r$. Given the QNM frequencies and the corresponding angular eigenvalues $A_{l m}$ it is a simple matter to compute $R_{r_{+}}$for any finite, not very large value of $r$. Unfortunately, the convergence of the Jaffé expansion gets worse for large values of $r$. This is precisely the region where the wavefunction can be expressed as a sum of in- and out-going components, a necessary procedure to evaluate $A_{\text {in }}^{T}$ and $A_{\text {out }}^{T}$ and their derivatives. For this reason we must resort to the Coulomb wavefunction representation first introduced by Leaver [12, 13], and summarized below.

\section{Coulomb wavefunction expansion of the radial wavefunction}

Let us first introduce a new wavefunction $h$, related to the Teukolsky wavefunction $R$ by

$$
R=\left(r-r_{-}\right)^{-1+i \sigma_{+}}\left(r-r_{+}\right)^{-s-i \sigma_{+}} h(z) .
$$

where $z \equiv \omega\left(r-r_{-}\right)$. The wavefunction $h$ satisfies the generalized spheroidal wave equation

$$
z\left(z-\omega x_{0}\right)\left[\partial_{z}^{2} h+(1-2 \eta / z) h\right]+C_{1} \omega \partial_{z} h+\left(C_{2}+C_{3} \omega / z\right) h=0,
$$

with

$$
\begin{aligned}
& x_{0}=b, \quad \eta=-i s-\omega \\
& C_{1}=b\left(1-s-2 i \sigma_{+}\right) \\
& C_{2}=-A_{l m}-s(s+1)+i b s \omega+2 \omega^{2}-a^{2} \omega^{2}+b \omega^{2} \\
& C_{3}=b(-1+s+i \omega)\left(1+i \omega-2 i \sigma_{+}\right) .
\end{aligned}
$$


We next expand $h$ as

$$
h(z)=\sum_{L=-\infty}^{\infty} a_{L} \mathcal{U}_{L+\nu}(\eta, z)
$$

where $\mathcal{U}_{L+\nu}(\eta, z)$ is any combination of the special functions $F_{L+\nu}(\eta, z)$ and $G_{L+\nu}(\eta, z)$, known as Coulomb wavefunctions (see [12, 52] for more details on these functions). We are interested in two particular combinations of Coulomb wavefunctions:

$$
\begin{aligned}
& \mathcal{U}_{L+\nu}^{+}(\eta, z) \equiv G_{L+\nu}(\eta, z)+i F_{L+\nu}(\eta, z) \\
& \mathcal{U}_{L+\nu}^{-}(\eta, z) \equiv G_{L+\nu}(\eta, z)-i F_{L+\nu}(\eta, z)
\end{aligned}
$$

As $z \rightarrow \infty$ these functions have a simple asymptotic behavior [52]:

$$
\mathcal{U}_{L+\nu}^{ \pm}(\eta, z) \sim\left(1 \pm \frac{(i \eta-L)(i \eta+L+1)}{2 i z}\right) e^{ \pm i\left(z-\eta \log 2 z-L \pi / 2+\sigma_{L}\right)}
$$

with

$$
\sigma_{L}=-\frac{i}{2} \log \frac{\Gamma(L+\nu+1+i \eta)}{\Gamma(L+\nu+1-i \eta)}
$$

Other properties of these functions are given in Appendix B4, The coefficients $a_{L}$ in (B25) are determined by

$$
\alpha_{L} a_{L+1}+\beta_{L} a_{L}+\gamma_{L} a_{L-1}=0
$$

where

$$
\begin{aligned}
& \alpha_{L}=-\frac{\omega \sqrt{(L+1+\nu)^{2}+\eta^{2}}}{(L+1+\nu)(2 L+2 \nu+3)}\left[(L+\nu+1)(L+\nu+2) x_{0}-C_{1}(L+\nu+2)-C_{3}\right], \\
& \beta_{L}=(L+\nu)(L+\nu+1)+C_{2}+\frac{\omega \eta}{(L+\nu)(L+\nu+1)}\left[(L+\nu)(L+\nu+1) x_{0}-C_{1}-C_{3}\right], \\
& \gamma_{L}=-\frac{\omega \sqrt{(L+\nu)^{2}+\eta^{2}}}{(L+\nu)(2 L+2 \nu-1)}\left[(L+\nu)(L+\nu-1) x_{0}-C_{1}(L+\nu-1)-C_{3}\right] .
\end{aligned}
$$

It is convenient to define the quantities 53 .

$$
R_{L} \equiv \frac{a_{L}}{a_{L-1}}, \quad L_{L} \equiv \frac{a_{L}}{a_{L+1}}
$$

From these definitions it follows that

$$
R_{L}=-\frac{\gamma_{L}}{\beta_{L}+\alpha_{L} R_{L+1}}, \quad L_{L}=-\frac{\alpha_{L}}{\beta_{L}+\gamma_{L} L_{L-1}} .
$$

For a minimal solution of the recurrence relation the (as yet undetermined) parameter $\nu$ must be a root of [12]

$$
\beta_{0}=\frac{\alpha_{-1} \gamma_{0}}{\beta_{-1}-} \frac{\alpha_{-2} \gamma_{-1}}{\beta_{-2}-} \frac{\alpha_{-3} \gamma_{-2}}{\beta_{-3}-} \ldots+\frac{\alpha_{0} \gamma_{1}}{\beta_{1}-} \frac{\alpha_{1} \gamma_{2}}{\beta_{2}-} \frac{\alpha_{2} \gamma_{3}}{\beta_{3}-} \ldots
$$

The parameter $\nu$ can also be determined as a solution of the equation [53]

$$
R_{L} L_{L-1}=1
$$

However, as suggested in [54], the most convenient numerical choice is to solve for

$$
\beta_{L}+\alpha_{L} R_{L+1}+\gamma_{L} L_{L-1}=0
$$

We are interested only in the solutions that map to the correct asymptotic value $\nu=l$ as $\omega \rightarrow 0$ (corrections of order $\omega^{2}$ can be found in [53]). Roots $\nu$ that are integer multiples of $1 / 2$ are usually spurious and must be discarded [12]. For slowly damped modes, physically meaningful roots are most easily obtained setting $L=0$ or $L=-1$ in (B38). 
We verified that different numerical procedures yield excellent agreement on the resulting values of $\nu$. For reference, we list some of these values along with the corresponding QNM frequencies (for $a=0$ ) in Table VII]

The procedure to compute the functions $R_{\infty_{+}}$and $R_{\infty_{-}}$is now straightforward, at least in principle. Compute the parameter $\nu$ solving (B36), (B37) or (B38). Then compute the coefficients $a_{L}$ for positive and negative $L$ from (B34), setting for instance $a_{0}=1$ (the final results are of course unaffected by this or other normalization choices). Finally, the general solution follows from (B19) and (B25). Imposing the normalization condition (4.8b) we get

$$
R_{\infty_{ \pm}}=\frac{\left(r-r_{-}\right)^{-1+i \sigma_{+}}\left(r-r_{+}\right)^{-s-i \sigma_{+}}}{K_{ \pm}} \sum_{L=-\infty}^{\infty} a_{L} \mathcal{U}_{L+\nu}^{ \pm}
$$

where from (B28) the normalization constants read

$$
K_{ \pm}=\sum_{L=-\infty}^{\infty} a_{L} e^{ \pm\left[-i \eta \log (2 \omega)-i(L+\nu) \pi / 2+i \sigma_{L}\right]}
$$

The nontrivial step in the calculation of (B39) is the evaluation of the Coulomb wavefunctions $\mathcal{U}_{L+\nu}^{ \pm}$for complex arguments. Computing these functions is particularly tricky for large values of $|L|$, which must be included for (B39) to converge with acceptable accuracy. Typically a good precision (of the order of five or more significant digits) is achieved summing terms up to $|L| \simeq 15$.

We verified our results in a number of ways. We first computed the functions $\mathcal{U}_{L+\nu}^{ \pm}(\eta, z)$ using their relation with the confluent hypergeometric function $U$, Eq. (125) in [12]. Then we checked their values using a similar representation in terms of the confluent hypergeometric function $M$, that can be derived from the integral representation (111) of [12]. The reason for switching to $U$ and $M$ is that confluent hypergeometric functions (unlike Coulomb wavefunctions) are implemented in the present version of Mathematica. Our Mathematica calculations agree very well with a Fortran subroutine to compute Coulomb wavefunctions for complex arguments [55], that was eventually used to obtain the results in this paper. Further checks on our numerical results are discussed in the following section.

\section{Numerical checks of the solutions: some useful identities}

Here we describe a number of consistency checks we performed on our numerical solutions. We performed the calculations using both Mathematica and a Fortran code. Our final results on the QNEFs typically agree to one part in $10^{4}$ or better.

The convergence of the series expansion (B8) was checked via a direct high-precision integration of the Teukolsky equation. When evaluated in the matching region (typically we use $r \sim 5-7$ ) the two methods agree to one part in $10^{7}$ or better.

Besides checking the validity of the asymptotic expansion (B28), the following equalities proved useful to determine the accuracy of the functions $\mathcal{U}_{ \pm}^{L+\nu}$ :

$$
\begin{aligned}
& \frac{d^{2} \mathcal{U}_{L+\nu}^{ \pm}}{d z^{2}}+\left(1-\frac{2 \eta}{z}-\frac{(L+\nu)(L+\nu+1)}{z^{2}}\right) \mathcal{U}_{L+\nu}^{ \pm}=0 \\
& \mathcal{U}_{L+\nu-1}^{+} \mathcal{U}_{L+\nu}^{-}-\mathcal{U}_{L+\nu}^{+} \mathcal{U}_{L+\nu-1}^{-}=\frac{2 i(L+\nu)}{\sqrt{(L+\nu)^{2}+\eta^{2}}} \\
& (L+\nu) \sqrt{(L+\nu+1)^{2}+\eta^{2}} \mathcal{U}_{L+\nu+1}^{ \pm}=(2(L+\nu)+1)\left(\eta+\frac{(L+\nu)(L+\nu+1)}{z}\right) \mathcal{U}_{L+\nu}^{ \pm}- \\
& (L+\nu+1) \sqrt{(L+\nu)^{2}+\eta^{2}} \mathcal{U}_{L+\nu-1}^{ \pm} \\
& \frac{d \mathcal{U}_{L+\nu}^{+}}{d z} \mathcal{U}_{L+\nu}^{-}-\mathcal{U}_{L+\nu}^{+} \frac{d \mathcal{U}_{L+\nu-1}^{-}}{d z}=2 i \\
& (L+\nu) \frac{d \mathcal{U}_{L+\nu}^{ \pm}=\sqrt{(L+\nu)^{2}+\eta^{2}} \mathcal{U}_{L+\nu-1}^{ \pm}-\left(\frac{(L+\nu)^{2}}{z}+\eta\right) \mathcal{U}_{L+\nu}^{ \pm}}{z}=\frac{d \mathcal{U}_{L+\nu}^{ \pm}}{d z}=\left(\frac{(L+\nu+1)^{2}}{z}+\eta\right) \mathcal{U}_{L+\nu}^{ \pm}-\sqrt{(L+\nu+1)^{2}+\eta^{2}} \mathcal{U}_{L+\nu+1}^{ \pm} \\
& (L+\nu+1) \frac{L^{ \pm}}{d z}
\end{aligned}
$$

Numerical calculations of $\mathcal{U}_{ \pm}^{L+\nu}$ usually fail for large $|L|$. This is true both when we use their representation in terms of confluent hypergeometrics and when we use the Fortran routines of Ref. [55]. In the sum (B39) we included only those values of $L$ for which the identity (B42) is satisfied (in modulus) to better than one part in $10^{4}$. 
An important test on the linear combinations $R_{\infty_{ \pm}}$is based on the calculation of their Wronskian. One can prove easily that the Wronskian $W$ between any two solutions of the Teukolsky equation satisfies

$$
W(r)=W\left(r_{0}\right) \exp \left[-\int_{r_{0}}^{r} \frac{(s+1)(2 x-1)}{\left(x-r_{+}\right)\left(x-r_{-}\right)} d x\right],
$$

where $r_{0}$ is any fixed point. Considering the two solutions $R_{\infty_{+}}$and $R_{\infty_{-}}$we can evaluate the constant $W\left(r_{0}\right)$ by computing the Wronskian at infinity from their asymptotic behavior (4.8b), with the result

$$
W\left(r_{0}\right)=2 i \omega\left[\left(r_{0}-r_{+}\right)\left(r_{0}-r_{-}\right)\right]^{-(s+1)} .
$$

Our wavefunctions satisfy this relation at different selected values of $r_{0}$. A final test consists in evaluating $A_{\text {out }}$ by a direct integration of the Teukolsky equation and comparing the results with the method described in the main text: the agreement is at the level of one percent or better.

\section{APPENDIX C: TRANSFORMATION BETWEEN THE TEUKOLSKY AND SASAKI-NAKAMURA WAVEFUNCTIONS}

In the main text we computed the QNEFs using the Teukolsky formalism. Quite often it is computationally convenient to use the equivalent formalism developed for $s=-2$ by Sasaki and Nakamura [22, 23, 24] and its generalization for other spins [25]. In general, switching between the two formalisms is not trivial. Fortunately, deriving the asymptotic behavior of the respective wavefunctions and the relation between the QNEFs is quite simple, as we show below.

We follow Sasaki and Tagoshi [24] and denote the SN function by $X^{(s)}$. At infinity, the asymptotic behavior of $X^{(s)}$ is the same for all $s$ :

$$
\lim _{r \rightarrow \infty} X^{(s)} \sim A_{\text {in }} e^{-i \omega r_{*}}+A_{\text {out }} e^{i \omega r_{*}},
$$

The normalization at the horizon is fixed by our choice for the Teukolsky function, Eqs. (4.7a) and (B8). We will define the QNEFs in the SN formalism in the usual way:

$$
B^{(s)}=\left.\frac{A_{\text {out }}}{2 \omega}\left(\frac{d A_{\text {in }}}{d \omega}\right)^{-1}\right|_{\omega=\omega_{n}} .
$$

Below we establish the relation between $B^{(s)}$ and the QNEFs $B_{T}^{(s)}$ in the Teukolsky formalism, as computed in the main text.

\section{Scalar perturbations}

In the scalar case, the generalized $\mathrm{SN}$ function is related to the Teukolsky radial function $R$ by [25]

$$
X^{(0)}=\left(r^{2}+a^{2}\right)^{1 / 2} R,
$$

and satisfies

$$
\frac{d^{2}}{d r_{*}^{2}} X^{(0)}-\mathcal{U}^{(0)} X^{(0)}=0
$$

where

$$
\mathcal{U}^{(0)}=-\frac{K^{2}-\left(A_{l m}-2 a m \omega+a^{2} \omega^{2}\right) \Delta}{\left(r^{2}+a^{2}\right)^{2}}+G^{2}+\frac{d}{d r_{*}} G,
$$

with $K=\left(r^{2}+a^{2}\right) \omega-a m$, and $G=r \Delta\left(r^{2}+a^{2}\right)^{-2}$.

From Eq. (C3) we have immediately

$$
B_{\mathrm{T}}^{(0)}=B^{(0)} .
$$

The normalization of the Teukolsky function, Eq. (4.7a), implies that at the horizon

$$
\lim _{r \rightarrow r_{+}} X^{(0)} \sim\left(r_{+}^{2}+a^{2}\right)^{1 / 2}\left(r_{+}-r_{-}\right)^{-1+i \omega+i \sigma_{+}} e^{i \omega r_{+}}\left(r-r_{+}\right)^{-i \sigma_{+}} .
$$




\section{Electromagnetic perturbations}

For the electromagnetic case, the generalized SN transformation is

$$
X^{(-1)}=\sqrt{\frac{r^{2}+a^{2}}{\Delta}}\left(\alpha R^{(-1)}+\beta \partial_{r} R^{(-1)}\right),
$$

where we can choose 25]

$$
\alpha=-\frac{r^{2}+a^{2}}{r^{2}} \sqrt{\Delta}\left(\frac{r}{r^{2}+a^{2}}+\frac{i K}{\Delta}\right), \quad \beta=\frac{r^{2}+a^{2}}{r^{2}} \sqrt{\Delta} .
$$

From the asymptotic behavior of the Teukolsky function

$$
\lim _{r \rightarrow \infty} R^{(-1)} \sim A_{\mathrm{in}}^{\mathrm{T}} r^{-1} e^{-i \omega r_{*}}\left(1+\mu_{1} / r\right)+A_{\mathrm{out}}^{\mathrm{T}} r e^{i \omega r_{*}}\left(1+\mu_{2} / r\right)
$$

with $\mu_{1}=\left[-2 \omega+i\left(2-A_{l m}-a^{2} \omega^{2}\right)\right] /(2 \omega)$ and $\mu_{2}=\left[i\left(A_{l m}+a^{2} \omega^{2}\right)\right] /(2 \omega)$ we get

$$
A_{\mathrm{in}}^{\mathrm{T}}=-\frac{1}{2 i \omega} A_{\mathrm{in}}, \quad A_{\mathrm{out}}^{\mathrm{T}}=-\frac{2 i \omega}{2 a m \omega-A_{l m}-a^{2} \omega^{2}} A_{\mathrm{out}},
$$

and finally

$$
B_{\mathrm{T}}^{(-1)}=-\frac{4 \omega^{2}}{2 a m \omega-A_{l m}-a^{2} \omega^{2}} B^{(-1)} .
$$

Similarly, from the asymptotic behavior of the Teukolsky function at the horizon (4.7a) we find

$$
\lim _{r \rightarrow r_{+}} X^{(-1)}=\left(1-2 i \sigma_{+}\right) r_{+}^{-1 / 2}\left(r_{+}-r_{-}\right)^{i \omega+i \sigma_{+}} e^{i \omega r_{+}}\left(r-r_{+}\right)^{-i \sigma_{+}} .
$$

\section{Gravitational perturbations}

For the gravitational case the transformation between the SN and Teukolsky wavefunctions is more complex, but here we only need the asymptotic behavior (for more details we refer to the original papers [22, 23] and to the review in [24]). One can show, eg. from Eqs. (65)-(69) in [24], that asymptotically the Teukolsky and SN amplitudes satisfy

$$
A_{\mathrm{in}}^{\mathrm{T}}=-\frac{1}{4 \omega^{2}} A_{\mathrm{in}}, \quad A_{\mathrm{out}}^{\mathrm{T}}=-\frac{4 \omega^{2}}{\lambda(\lambda+2)-6 i \omega-12 a \omega(a \omega-m)} A_{\mathrm{out}},
$$

so that

$$
B_{\mathrm{T}}^{(-2)}=\frac{16 \omega^{4}}{\lambda(\lambda+2)-6 i \omega-12 a \omega(a \omega-m)} B^{(-2)} .
$$

with $\lambda \equiv A_{l m}+(a \omega)^{2}-2 a m \omega$. Expressing Eqs. (65) and (68) in [24] in Leaver's units and combining them with Eq. (4.7a), the normalization at the horizon is:

$$
\lim _{r \rightarrow r_{+}} X^{(-2)}=d\left(r_{+}-r_{-}\right)^{-1+i \omega+i \sigma_{+}} e^{i \omega r_{+}}\left(r-r_{+}\right)^{-i \sigma_{+}}
$$

where $d \equiv r_{+}^{1 / 2}\left[\left(8-12 i \omega-4 \omega^{2}\right) r_{+}^{2}+(12 i a m-8+8 a m \omega+6 i \omega) r_{+}-4 a^{2} m^{2}-6 i a m+2\right]$.

[1] J. S. B. Wyithe, A. Loeb, Astrophys. J. 612, 597 (2004).

[2] K. Kulczycki, T. Bulik, K. Belczynski, B. Rudak, arXiv:astro-ph/0602533.

[3] E. E. Flanagan and S. A. Hughes, Phys. Rev. D 57, 4535 (1998).

[4] E. Berti, V. Cardoso and C. M. Will, Phys. Rev. D 73, 064030 (2006). 
[5] E. Berti, Class. Quantum Grav. 23, S785 (2006).

[6] O. Dreyer, B. Kelly, B. Krishnan, L. S. Finn, D. Garrison and R. Lopez-Aleman, Class. Quantum Grav. 21, 787 (2004).

[7] E. Berti and V. Cardoso, arXiv:gr-qc/0605101.

[8] E. Berti, V. Cardoso and M. Casals, Phys. Rev. D 73, 024013 (2006).

[9] E. Berti, V. Cardoso and C. M. Will, arXiv:gr-qc/0601077.

[10] C. V. Vishveshwara, Nature (London) 227, 936 (1970).

[11] E. W. Leaver, Proc. R. Soc. London A402, 285 (1985).

[12] E. W. Leaver, J. Math. Phys. 27, 1238 (1986).

[13] E. W. Leaver, Phys. Rev. D 34, 384 (1986); Erratum-ibid. D 38, 725 (1988).

[14] Y. Sun and R. H. Price, Phys. Rev. D 38, 1040 (1988).

[15] H.-P. Nollert and B. G. Schmidt, Phys. Rev. D 45, 2617 (1992).

[16] H.-P. Nollert and R. H. Price, J. Math. Phys. 40, 980 (1999).

[17] N. Andersson, Phys. Rev. D 51, 353 (1995).

[18] N. Andersson, Phys. Rev. D 55, 468 (1997).

[19] K. Glampedakis and N. Andersson, Phys. Rev. D 64, 104021 (2001).

[20] K. Glampedakis and N. Andersson, Class. Quant. Grav. 20, 3441 (2003).

[21] P.M. Morse and H. Feshbach, Methods of Theoretical Physics, (McGraw Hill, New York, 1953).

[22] M. Sasaki and T. Nakamura, Phys. Lett. 87A, 85 (1981); Phys. Lett. 89A, 68 (1982).

[23] M. Sasaki and T. Nakamura, Prog. Theor. Phys. 67, 1788 (1982).

[24] M. Sasaki and H. Tagoshi, Living Rev. Rel. 6, 6 (2003). The angular separation constant $\lambda$ in this reference is the same as Teukolsky's [32] and tends to $l(l+1)-s(s+1)$ in the Schwarzschild limit, contrary to the claim below Eq. (18).

[25] S. A. Hughes, Phys. Rev. D 62, 044029 (2000); Erratum-ibid. D 67, 089902 (2003).

[26] In Eq. (2.4) of Sec. II the potential $V(x)$ and the source $\mathcal{S}$ were introduced to reproduce the structure of Eq. (3.1). In general, in the $\mathrm{SN}$ formalism the wave equation contains a first derivative of the wavefunction:

$$
\frac{d^{2} X}{d r_{*}^{2}}-\mathcal{F} \frac{d X}{d r_{*}}-\mathcal{U} X=S
$$

where we also allow for the presence of a source term $S$. This is no restriction, since a trivial change of variable of the form

$$
X=\exp \left[\int \frac{\mathcal{F}}{2} d r_{*}\right] X_{2}
$$

eliminates the first derivative, yielding

$$
\frac{d^{2} X_{2}}{d r_{*}^{2}}+\left(\frac{\mathcal{F}^{\prime}}{2}-\frac{\mathcal{F}^{2}}{4}-\mathcal{U}\right) X_{2}=S \exp \left[-\int \frac{\mathcal{F}}{2} d r_{*}\right] .
$$

[27] K. D. Kokkotas and B. G. Schmidt, Living Rev. Rel. 2, 2 (1999); H.-P. Nollert, Class. Quantum Grav. 16, R159 (1999).

[28] R. H. Price, Phys. Rev. D 5, 2419 (1972).

[29] H.-P. Nollert, Characteristic Oscillations of Black Holes and Neutron Stars: From Mathematical Background to Astrophysical Applications, Habilitationsschrift Der Fakultät für Physik der Eberhard-Karls-Universität, Tübingen (2000).

[30] B. P. Jensen and P. Candelas, Phys. Rev. D 33, 1590 (1986); Erratum ibidem 35, 4041 (1987).

[31] E. S. C. Ching, P. T. Leung, W. M. Suen and K. Young, Phys. Rev. Lett. 74, 2414 (1995); idem, Phys. Rev. D 52, 2118 (1995).

[32] S. A. Teukolsky, Astrophys. J. 185, 635 (1973).

[33] H. Onozawa, Phys. Rev. D 55, 3593 (1997); E. Berti and K. D. Kokkotas, Phys. Rev. D 68, 044027 (2003); E. Berti, V. Cardoso, K. D. Kokkotas and H. Onozawa, Phys. Rev. D 68, 124018 (2003); E. Berti, V. Cardoso and S. Yoshida, Phys. Rev. D 69, 124018 (2004).

[34] The main source of disagreement seems to be a bug in the computer code used in [20] to compute scalar QNEFs of Kerr black holes (N. Andersson and K. Glampedakis, private communication).

[35] N. Andersson, private communication.

[36] V. Ferrari and B. Mashhoon, Phys. Rev. D 30, 295 (1984).

[37] E. Berti, arXiv:gr-qc/0411025

[38] E. Berti and K. D. Kokkotas, Phys. Rev. D 71, 124008 (2005).

[39] S. Chandrasekhar, The Mathematical Theory of Black Holes (Oxford University, New York, 1983).

[40] E. N. Dorband, E. Berti, P. Diener, E. Schnetter and M. Tiglio, arXiv:gr-qc/0608091.

[41] Y. Zlochower, R. Gomez, S. Husa, L. Lehner and J. Winicour, Phys. Rev. D 68, 084014 (2003).

[42] M. Campanelli, B. J. Kelly and C. O. Lousto, Phys. Rev. D 73, 064005 (2006).

[43] J. G. Baker, J. Centrella, D. I. Choi, M. Koppitz, J. R. van Meter and M. C. Miller, arXiv:astro-ph/0603204

[44] T. Damour and A. Gopakumar, Phys. Rev. D 73, 124006 (2006).

[45] L. Motl and A. Neitzke, Adv. Theor. Math. Phys.7, 307 (2003).

[46] V. Cardoso, J. P. S. Lemos and S. Yoshida, Phys. Rev. D 69, 044004 (2004).

[47] A. Neitzke, arXiv:hep-th/0304080. 
TABLE I: QNM frequencies of the first two overtones with $s=0$ and $l=2$, for several values of rotation parameter and for some values of $m$. For consistency with [4], with Table 3 of [20] and with most of the QNM literature, here we depart from Leaver's unit convention (but not from his Fourier transform convention) listing the dimensionless frequencies $\omega M$.

\begin{tabular}{cccccc}
\multicolumn{5}{c}{$s=0, n=0$} \\
\hline$j$ & $m=-2$ & $m=-1$ & $m=0$ & $m=1$ & $m=2$ \\
\hline 0.00 & $0.48364-0.09676 i$ & $0.48364-0.09676 i$ & $0.48364-0.09676 i$ & $0.48364-0.09676 i$ & $0.48364-0.09676 i$ \\
0.20 & $0.45620-0.09655 i$ & $0.47015-0.09650 i$ & $0.48491-0.09646 i$ & $0.50055-0.09641 i$ & $0.51712-0.09638 i$ \\
0.40 & $0.43306-0.09599 i$ & $0.45932-0.09577 i$ & $0.48886-0.09547 i$ & $0.52214-0.09515 i$ & $0.55964-0.09493 i$ \\
0.50 & $0.42275-0.09562 i$ & $0.45477-0.09523 i$ & $0.49196-0.09463 i$ & $0.53536-0.09397 i$ & $0.58599-0.09349 i$ \\
0.60 & $0.41315-0.09520 i$ & $0.45073-0.09457 i$ & $0.49594-0.09348 i$ & $0.55080-0.09219 i$ & $0.61737-0.09125 i$ \\
0.80 & $0.39573-0.09429 i$ & $0.44407-0.09280 i$ & $0.50713-0.08967 i$ & $0.59202-0.08513 i$ & $0.70683-0.08152 i$ \\
0.90 & $0.38780-0.09379 i$ & $0.44141-0.09165 i$ & $0.51478-0.08641 i$ & $0.62183-0.07718 i$ & $0.78164-0.06929 i$ \\
0.98 & $0.38177-0.09338 i$ & $0.43957-0.09057 i$ & $0.52212-0.08254 i$ & $0.65470-0.06290 i$ & $0.89802-0.04090 i$ \\
\hline \hline
\end{tabular}

\begin{tabular}{cccccc}
\multicolumn{5}{c}{$s=0, n=1$} \\
\hline$j$ & $m=-2$ & $m=-1$ & $m=0$ & $m=1$ & $m=2$ \\
\hline 0.00 & $0.46385-0.29560 i$ & $0.46385-0.29560 i$ & $0.46385-0.29560 i$ & $0.46385-0.29560 i$ & $0.46385-0.29560 i$ \\
0.20 & $0.43418-0.29576 i$ & $0.44940-0.29518 i$ & $0.46539-0.29460 i$ & $0.48221-0.29405 i$ & $0.49989-0.29358 i$ \\
0.40 & $0.40907-0.29477 i$ & $0.43805-0.29314 i$ & $0.47013-0.29132 i$ & $0.50578-0.28955 i$ & $0.54541-0.28821 i$ \\
0.50 & $0.39787-0.29398 i$ & $0.43337-0.29153 i$ & $0.47381-0.28856 i$ & $0.52020-0.28555 i$ & $0.57344-0.28334 i$ \\
0.60 & $0.38740-0.29304 i$ & $0.42927-0.28950 i$ & $0.47843-0.28476 i$ & $0.53697-0.27969 i$ & $0.60667-0.27599 i$ \\
0.80 & $0.36839-0.29081 i$ & $0.42253-0.28395 i$ & $0.49060-0.27224 i$ & $0.58067-0.25688 i$ & $0.70042-0.24548 i$ \\
0.90 & $0.35971-0.28956 i$ & $0.41969-0.28029 i$ & $0.49759-0.26177 i$ & $0.60984-0.23150 i$ & $0.77768-0.20801 i$ \\
0.98 & $0.35310-0.28851 i$ & $0.41754-0.27687 i$ & $0.50230-0.25024 i$ & $0.62982-0.18739 i$ & $0.89622-0.12214 i$ \\
\hline \hline
\end{tabular}

[48] V. Cardoso, J. Natário and R. Schiappa, J. Math. Phys. 45, 4698 (2004); J. Natário and R. Schiappa, Adv. Theor. Math. Phys. 8, 1001 (2004); H. Nomura and T. Tamaki, Phys. Rev. D 71, 124033 (2005); R. G. Daghigh and G. Kunstatter, Class. Quant. Grav. 22, 4113 (2005); arXiv:gr-qc/0507019, A. Ghosh, S. Shankaranarayanan and S. Das, Class. Quant. Grav. 23, 1851 (2006).

[49] H. R. Beyer, Commun. Math. Phys. 204, 397 (1999); V. Cardoso and J. P. S. Lemos, Phys. Rev. D 67, 084020 (2003); A. Maassen van den Brink, Phys. Rev. D 68, 047501 (2003).

[50] A. J. M. Medved, D. Martin and M. Visser, Class. Quant. Grav. 21, 1393 (2004).

[51] S. Chandrasekhar and S. Detweiler, Proc. Roy. Soc. London, Series A 344, 441 (1975).

[52] M. Abramowitz and I. A. Stegun, Handbook of Mathematical Functions, (Dover, New York, 1970).

[53] S. Mano, H. Suzuki and E. Takasugi, Progr. Theor. Phys. 95, 1079 (1996).

[54] R. Fujita and H. Tagoshi, Prog. Theor. Phys. 112, 415 (2004).

[55] I. J. Thompson and A. R. Barnett, Comp. Physics Commun. 36, 363 (1985); Erratum-ibid. 159, 241 (2004); I. J. Thompson and A. R. Barnett, Journal of Computational Physics 64, 490 (1986). 
TABLE II: Angular separation constants $A_{l m}$ of the first two overtones with $s=0$ and $l=2$, for several values of rotation parameter and for some values of $m$.

\begin{tabular}{cccccc}
\multicolumn{5}{c}{$s=0, n=0$} \\
\hline$j$ & $m=-2$ & $m=-1$ & $m=0$ & $m=1$ & $m=2$ \\
\hline 0.00 & $6.0000+0.00000 i$ & $6.0000+0.00000 i$ & $6.0000+0.00000 i$ & $6.0000+0.00000 i$ & $6.0000+0.00000 i$ \\
0.20 & $5.9989+0.00050 i$ & $5.9964+0.00156 i$ & $5.9953+0.00196 i$ & $5.9959+0.00166 i$ & $5.9985+0.00057 i$ \\
0.40 & $5.9959+0.00190 i$ & $5.9862+0.00604 i$ & $5.9807+0.00781 i$ & $5.9819+0.00682 i$ & $5.9930+0.00243 i$ \\
0.50 & $5.9939+0.00289 i$ & $5.9788+0.00929 i$ & $5.9695+0.01217 i$ & $5.9702+0.01080 i$ & $5.9880+0.00392 i$ \\
0.60 & $5.9917+0.00405 i$ & $5.9700+0.01317 i$ & $5.9553+0.01743 i$ & $5.9544+0.01570 i$ & $5.9808+0.00582 i$ \\
0.80 & $5.9865+0.00684 i$ & $5.9482+0.02266 i$ & $5.9167+0.03030 i$ & $5.9056+0.02776 i$ & $5.9547+0.01063 i$ \\
0.90 & $5.9836+0.00845 i$ & $5.9352+0.02817 i$ & $5.8911+0.03744 i$ & $5.8674+0.03351 i$ & $5.9294+0.01271 i$ \\
0.98 & $5.9812+0.00982 i$ & $5.9237+0.03289 i$ & $5.8668+0.04293 i$ & $5.8245+0.03416 i$ & $5.8884+0.01030 i$ \\
\hline \hline
\end{tabular}

\begin{tabular}{cccccc}
\multicolumn{5}{c}{$s=0, n=1$} \\
\hline$j$ & $m=-2$ & $m=-1$ & $m=0$ & $m=1$ & $m=2$ \\
\hline 0.00 & $6.0000+0.00000 i$ & $6.0000+0.00000 i$ & $6.0000+0.00000 i$ & $6.0000+0.00000 i$ & $6.0000+0.00000 i$ \\
0.20 & $5.9994+0.00147 i$ & $5.9980+0.00455 i$ & $5.9973+0.00575 i$ & $5.9975+0.00486 i$ & $5.9991+0.00168 i$ \\
0.40 & $5.9982+0.00552 i$ & $5.9927+0.01762 i$ & $5.9886+0.02294 i$ & $5.9882+0.02010 i$ & $5.9951+0.00719 i$ \\
0.50 & $5.9974+0.00836 i$ & $5.9890+0.02709 i$ & $5.9815+0.03577 i$ & $5.9797+0.03186 i$ & $5.9911+0.01163 i$ \\
0.60 & $5.9967+0.01169 i$ & $5.9845+0.03838 i$ & $5.9720+0.05129 i$ & $5.9676+0.04641 i$ & $5.9850+0.01727 i$ \\
0.80 & $5.9954+0.01961 i$ & $5.9732+0.06591 i$ & $5.9439+0.08920 i$ & $5.9256+0.08210 i$ & $5.9606+0.03168 i$ \\
0.90 & $5.9948+0.02414 i$ & $5.9662+0.08181 i$ & $5.9237+0.10993 i$ & $5.8894+0.09850 i$ & $5.9347+0.03791 i$ \\
0.98 & $5.9944+0.02799 i$ & $5.9599+0.09535 i$ & $5.9043+0.12559 i$ & $5.8509+0.09778 i$ & $5.8908+0.03067 i$ \\
\hline \hline
\end{tabular}

TABLE III: QNEFs $B^{(0)}$ of the first two overtones with $s=0$ and $l=2$, for several values of rotation parameter and for some values of $m$.

\begin{tabular}{cccccc}
\multicolumn{5}{c}{$s=0, n=0$} \\
\hline$j$ & $m=-2$ & $m=-1$ & $m=0$ & $m=1$ & $m=2$ \\
\hline 0.00 & $0.11936+0.01343 i$ & $0.11936+0.01343 i$ & $0.11936+0.01343 i$ & $0.11936+0.01343 i$ & $0.11936+0.01343 i$ \\
0.20 & $0.12028-0.00281 i$ & $0.12013+0.00695 i$ & $0.11907+0.01746 i$ & $0.11687+0.02868 i$ & $0.11332+0.04050 i$ \\
0.40 & $0.12079-0.01047 i$ & $0.12090+0.00828 i$ & $0.11714+0.03012 i$ & $0.10769+0.05442 i$ & $0.09045+0.07951 i$ \\
0.50 & $0.12156-0.01146 i$ & $0.12130+0.01197 i$ & $0.11447+0.04016 i$ & $0.09715+0.07141 i$ & $0.06518+0.10076 i$ \\
0.60 & $0.12283-0.01049 i$ & $0.12149+0.01794 i$ & $0.10945+0.05296 i$ & $0.07919+0.09011 i$ & $0.02441+0.11621 i$ \\
0.80 & $0.12720-0.00092 i$ & $0.11939+0.03899 i$ & $0.08459+0.08650 i$ & $0.00372+0.11379 i$ & $-0.09556+0.05169 i$ \\
0.90 & $0.13027+0.01075 i$ & $0.11456+0.05709 i$ & $0.05691+0.10467 i$ & $-0.05609+0.08585 i$ & $-0.05739-0.07009 i$ \\
0.98 & $0.13253+0.03151 i$ & $0.10313+0.08251 i$ & $0.01644+0.11542 i$ & $-0.07590+0.01089 i$ & $0.03015+0.03247 i$ \\
\hline \hline
\end{tabular}

\begin{tabular}{cccccc}
\multicolumn{5}{c}{$s=0, n=1$} \\
\hline$j$ & $m=-2$ & $m=-1$ & $m=0$ & $m=1$ & $m=2$ \\
\hline 0.00 & $0.03552-0.26427 i$ & $0.03552-0.26427 i$ & $0.03552-0.26427 i$ & $0.03552-0.26427 i$ & $0.03552-0.26427 i$ \\
0.20 & $0.00031-0.23879 i$ & $0.02062-0.25361 i$ & $0.04536-0.26760 i$ & $0.07508-0.27998 i$ & $0.11026-0.28969 i$ \\
0.40 & $-0.01305-0.22194 i$ & $0.02373-0.25063 i$ & $0.07830-0.27535 i$ & $0.15482-0.28823 i$ & $0.25502-0.27656 i$ \\
0.50 & $-0.01418-0.21735 i$ & $0.03186-0.25195 i$ & $0.10651-0.27817 i$ & $0.21694-0.27760 i$ & $0.36021-0.21856 i$ \\
0.60 & $-0.01204-0.21552 i$ & $0.04520-0.25482 i$ & $0.14519-0.27668 i$ & $0.29610-0.24132 i$ & $0.46944-0.08037 i$ \\
0.80 & $0.00433-0.22175 i$ & $0.09487-0.26260 i$ & $0.26097-0.23285 i$ & $0.44182+0.01597 i$ & $0.24418+0.54878 i$ \\
0.90 & $0.02430-0.23258 i$ & $0.14156-0.26409 i$ & $0.33334-0.16103 i$ & $0.33715+0.26654 i$ & $-0.50193+0.31383 i$ \\
0.98 & $0.06291-0.25169 i$ & $0.21530-0.25651 i$ & $0.39147-0.04418 i$ & $0.06435+0.29230 i$ & $0.23752-0.12729 i$ \\
\hline \hline
\end{tabular}


TABLE IV: Amplitudes $A_{\text {out }}$ of the first two overtones with $s=0$ and $l=2$, for several values of rotation parameter and for some values of $(l, m)$.

\begin{tabular}{cccccc}
\multicolumn{5}{c}{$s=0, n=0$} \\
\hline$j$ & $m=-2$ & $m=-1$ & $m=0$ & $m=1$ & $m=2$ \\
\hline 0.00 & $1.1472-1.1426 i$ & $1.1472-1.1426 i$ & $1.1472-1.1426 i$ & $1.1472-1.1426 i$ & $1.1472-1.1426 i$ \\
0.20 & $1.1068-1.2514 i$ & $1.1252-1.2067 i$ & $1.1455-1.1601 i$ & $1.1677-1.1115 i$ & $1.1915-1.0608 i$ \\
0.40 & $1.0516-1.4122 i$ & $1.0888-1.3208 i$ & $1.1353-1.2211 i$ & $1.1904-1.1119 i$ & $1.2522-0.9921 i$ \\
0.50 & $1.0057-1.5269 i$ & $1.0547-1.4093 i$ & $1.1203-1.2776 i$ & $1.2013-1.1287 i$ & $1.2930-0.9595 i$ \\
0.60 & $0.9311-1.6802 i$ & $0.9955-1.5331 i$ & $1.0886-1.3636 i$ & $1.2085-1.1641 i$ & $1.3444-0.9272 i$ \\
0.80 & $0.5060-2.2157 i$ & $0.6304-1.9952 i$ & $0.8437-1.7289 i$ & $1.1526-1.3741 i$ & $1.5014-0.8727 i$ \\
0.90 & $-0.3226-2.7081 i$ & $-0.1229-2.4419 i$ & $0.2580-2.1461 i$ & $0.8860-1.7127 i$ & $1.6345-0.9057 i$ \\
0.98 & $-3.8026-2.0011 i$ & $-3.3378-1.6360 i$ & $-2.6005-1.6232 i$ & $-1.1381-2.0428 i$ & $1.7179-1.4791 i$ \\
\hline \hline
\end{tabular}

\begin{tabular}{cccccc}
\multicolumn{5}{c}{$s=0, n=1$} \\
\hline$j$ & $m=-2$ & $m=-1$ & $m=0$ & $m=1$ & $m=2$ \\
\hline 0.00 & $-2.9403+3.1839 i$ & $-2.9403+3.1839 i$ & $-2.9403+3.1839 i$ & $-2.9403+3.1839 i$ & $-2.9403+3.1839 i$ \\
0.20 & $-2.3498+2.9311 i$ & $-2.6009+3.0411 i$ & $-2.8990+3.1573 i$ & $-3.2543+3.2790 i$ & $-3.6792+3.4045 i$ \\
0.40 & $-1.8312+2.6818 i$ & $-2.2132+2.8695 i$ & $-2.7542+3.0781 i$ & $-3.5300+3.2999 i$ & $-4.6593+3.5080 i$ \\
0.50 & $-1.5742+2.5630 i$ & $-1.9831+2.7773 i$ & $-2.6206+3.0195 i$ & $-3.6326+3.2719 i$ & $-5.2745+3.4588 i$ \\
0.60 & $-1.3002+2.4481 i$ & $-1.7093+2.6818 i$ & $-2.4198+2.9493 i$ & $-3.6819+3.2125 i$ & $-5.9898+3.2826 i$ \\
0.80 & $-0.5767+2.2022 i$ & $-0.8741+2.4579 i$ & $-1.5934+2.7666 i$ & $-3.3396+2.9990 i$ & $-7.6627+2.1745 i$ \\
0.90 & $0.0721+1.9681 i$ & $-0.0536+2.2109 i$ & $-0.5925+2.5617 i$ & $-2.4246+2.8572 i$ & $-8.3413+0.9505 i$ \\
0.98 & $1.2103+0.9694 i$ & $1.3828+0.9603 i$ & $1.4079+1.1980 i$ & $0.4182+1.9828 i$ & $-8.0724+0.3919 i$ \\
\hline \hline
\end{tabular}


TABLE V: QNEFs $B^{(-2)}$ of the first two overtones with $s=-2$ and $l=2$, for several values of the rotation parameter and all values of $m$.

Sasaki-Nakamura, $s=-2, n=0$

\begin{tabular}{cccccc}
\hline$j$ & $m=-2$ & $m=-1$ & $m=0$ & $m=1$ & $m=2$ \\
\hline 0.00 & $0.12690+0.02032 i$ & $0.12690+0.02032 i$ & $0.12690+0.02032 i$ & $0.12690+0.02032 i$ & $0.12690+0.02032 i$ \\
0.20 & $0.13384+0.00380 i$ & $0.13066+0.01398 i$ & $0.12644+0.02431 i$ & $0.12114+0.03460 i$ & $0.11481+0.04465 i$ \\
0.40 & $0.13951-0.00575 i$ & $0.13409+0.01509 i$ & $0.12399+0.03694 i$ & $0.10873+0.05810 i$ & $0.089192+0.076810 i$ \\
0.50 & $0.14255-0.00798 i$ & $0.13574+0.01864 i$ & $0.12088+0.04702 i$ & $0.096848+0.073395 i$ & $0.066032+0.094125 i$ \\
0.60 & $0.14598-0.00838 i$ & $0.13716+0.02454 i$ & $0.11523+0.05998 i$ & $0.077845+0.090076 i$ & $0.03074+0.10817 i$ \\
0.80 & $0.15473-0.00179 i$ & $0.13753+0.04581 i$ & $0.08743+0.09424 i$ & $0.00070+0.10841 i$ & $-0.091113+0.061347 i$ \\
0.90 & $0.16055+0.00821 i$ & $0.13413+0.06426 i$ & $0.05603+0.11183 i$ & $-0.059511+0.072290 i$ & $-0.061104-0.089943 i$ \\
0.98 & $0.16652+0.02731 i$ & $0.12487+0.08994 i$ & $0.01200+0.11809 i$ & $-0.056299-0.016701 i$ & $-0.042248+0.067331 i$ \\
\hline \hline
\end{tabular}

\begin{tabular}{cccccc}
\multicolumn{5}{c}{ Teukolsky, $s=-2, n=0$} \\
\hline$j$ & $m=-2$ & $m=-1$ & $m=0$ & $m=1$ & $m=2$ \\
\hline 0.00 & $0.025587-0.016876 i$ & $0.025587-0.016876 i$ & $0.025587-0.016876 i$ & $0.025587-0.016876 i$ & $0.025587-0.016876 i$ \\
0.20 & $0.015858-0.015534 i$ & $0.020659-0.016165 i$ & $0.026663-0.016183 i$ & $0.034089-0.015199 i$ & $0.043137-0.012654 i$ \\
0.40 & $0.010593-0.013107 i$ & $0.018158-0.014516 i$ & $0.029957-0.013582 i$ & $0.047365-0.006739 i$ & $0.070428+0.012901 i$ \\
0.50 & $0.008973-0.011907 i$ & $0.017585-0.013461 i$ & $0.032435-0.011025 i$ & $0.055380+0.003377 i$ & $0.081892+0.046677 i$ \\
0.60 & $0.007817-0.010778 i$ & $0.017385-0.012229 i$ & $0.035338-0.007035 i$ & $0.062135+0.021050 i$ & $0.07301+0.10619 i$ \\
0.80 & $0.006570-0.008740 i$ & $0.017957-0.008866 i$ & $0.040011+0.008791 i$ & $0.034721+0.091829 i$ & $-0.24081+0.15010 i$ \\
0.90 & $0.006467-0.007753 i$ & $0.018690-0.006243 i$ & $0.037266+0.023214 i$ & $-0.05801+0.10686 i$ & $-0.13315-0.45394 i$ \\
0.98 & $0.006929-0.006758 i$ & $0.019607-0.002616 i$ & $0.025180+0.038227 i$ & $-0.10800-0.041437 i$ & $-0.60610+0.26215 i$ \\
\hline \hline
\end{tabular}

\begin{tabular}{cccccc}
\multicolumn{6}{c}{ Sasaki-Nakamura, $s=-2, n=1$} \\
\hline$j$ & $m=-2$ & $m=-1$ & $m=0$ & $m=1$ & $m=2$ \\
\hline 0.00 & $0.04769-0.22379 i$ & $0.04769-0.22379 i$ & $0.04769-0.22379 i$ & $0.04769-0.22379 i$ & $0.04769-0.22379 i$ \\
0.20 & $0.01275-0.21222 i$ & $0.03286-0.22075 i$ & $0.05588-0.22700 i$ & $0.08149-0.23024 i$ & $0.10888-0.22995 i$ \\
0.40 & $-0.00572-0.20420 i$ & $0.03229-0.22415 i$ & $0.08389-0.23496 i$ & $0.14621-0.22961 i$ & $0.20947-0.20386 i$ \\
0.50 & $-0.01064-0.20281 i$ & $0.03752-0.22859 i$ & $0.10852-0.23864 i$ & $0.19604-0.21680 i$ & $0.27571-0.15875 i$ \\
0.60 & $-0.01302-0.20359 i$ & $0.04716-0.23482 i$ & $0.14339-0.23886 i$ & $0.25927-0.18219 i$ & $0.34491-0.07025 i$ \\
0.80 & $-0.00904-0.21482 i$ & $0.08713-0.25154 i$ & $0.25286-0.19967 i$ & $0.36945+0.05388 i$ & $0.29993+0.40490 i$ \\
0.90 & $0.00093-0.22925 i$ & $0.12656-0.26018 i$ & $0.32055-0.12532 i$ & $0.23328+0.28598 i$ & $-0.55384+0.46277 i$ \\
0.98 & $0.02390-0.25495 i$ & $0.18872-0.26443 i$ & $0.35732-0.00915 i$ & $-0.08578+0.18065 i$ & $0.58139+0.37949 i$ \\
\hline \hline
\end{tabular}

\begin{tabular}{cccccc}
\multicolumn{5}{c}{ Teukolsky, $s=-2, n=1$} \\
\hline$j$ & $m=-2$ & $m=-1$ & $m=0$ & $m=1$ & $m=2$ \\
\hline 0.00 & $-0.081135+0.067726 i$ & $-0.081135+0.067726 i$ & $-0.081135+0.067726 i$ & $-0.081135+0.067726 i$ & $-0.081135+0.067726 i$ \\
0.20 & $-0.042052+0.057934 i$ & $-0.061317+0.063041 i$ & $-0.086857+0.065808 i$ & $-0.12023+0.06413 i$ & $-0.16306+0.05488 i$ \\
0.40 & $-0.023703+0.046853 i$ & $-0.053312+0.056703 i$ & $-0.10514+0.05697 i$ & $-0.18937+0.02718 i$ & $-0.30908-0.07767 i$ \\
0.50 & $-0.018645+0.042289 i$ & $-0.052550+0.053249 i$ & $-0.11959+0.04661 i$ & $-0.23424-0.02592 i$ & $-0.36754-0.28206 i$ \\
0.60 & $-0.015312+0.038467 i$ & $-0.053615+0.049396 i$ & $-0.13714+0.02823 i$ & $-0.27023-0.12799 i$ & $-0.26075-0.66420 i$ \\
0.80 & $-0.012449+0.033074 i$ & $-0.061191+0.038443 i$ & $-0.16317-0.05596 i$ & $-0.00912-0.52799 i$ & $2.1447-0.0490 i$ \\
0.90 & $-0.012978+0.031609 i$ & $-0.068071+0.029129 i$ & $-0.13659-0.13081 i$ & $0.60114-0.32896 i$ & $-2.2114+3.6692 i$ \\
0.98 & $-0.015950+0.031612 i$ & $-0.077430+0.015783 i$ & $-0.05976-0.18580 i$ & $0.14397+0.42835 i$ & $3.7992+4.8108 i$ \\
\hline \hline
\end{tabular}


TABLE VI: Amplitudes $A_{\text {out }}$ of the first two overtones with $s=-2$ and $l=2$, for several values of the rotation parameter and all values of $m$.

Sasaki-Nakamura, $s=-2, n=0$

\begin{tabular}{cccccc}
\hline$j$ & $m=-2$ & $m=-1$ & $m=0$ & $m=1$ & $m=2$ \\
\hline 0.00 & $-4.7608+2.3771 i$ & $-4.7608+2.3771 i$ & $-4.7608+2.3771 i$ & $-4.7608+2.3771 i$ & $-4.7608+2.3771 i$ \\
0.20 & $-6.2957+3.7664 i$ & $-5.4365+3.0202 i$ & $-4.6676+2.3909 i$ & $-3.9841+1.8690 i$ & $-3.3819+1.4446 i$ \\
0.40 & $-7.8741+5.8832 i$ & $-5.9349+3.8929 i$ & $-4.3699+2.4414 i$ & $-3.1373+1.4490 i$ & $-2.2068+0.8273 i$ \\
0.50 & $-8.5905+7.3807 i$ & $-6.0631+4.4740 i$ & $-4.1247+2.4901 i$ & $-2.6907+1.2641 i$ & $-1.7053+0.6084 i$ \\
0.60 & $-9.1362+9.3469 i$ & $-6.0428+5.2133 i$ & $-3.7910+2.5653 i$ & $-2.2271+1.0932 i$ & $-1.2657+0.4363 i$ \\
0.80 & $-8.311+16.035 i$ & $-4.8599+7.6211 i$ & $-2.6288+2.8690 i$ & $-1.2248+0.7843 i$ & $-0.58015+0.18762 i$ \\
0.90 & $-3.399+22.656 i$ & $-2.0780+9.8297 i$ & $-1.3270+3.1477 i$ & $-0.63687+0.62642 i$ & $-0.32078+0.07721 i$ \\
0.98 & $23.657+28.208 i$ & $9.6770+9.8318 i$ & $2.1270+2.4156 i$ & $0.03281+0.34446 i$ & $-0.09492-0.12174 i$ \\
\hline \hline
\end{tabular}

Teukolsky, $s=-2, n=0$

\begin{tabular}{cccccc}
\hline$j$ & $m=-2$ & $m=-1$ & $m=0$ & $m=1$ & $m=2$ \\
\hline 0.00 & $0.39793-0.36149 i$ & $0.39793-0.36149 i$ & $0.39793-0.36149 i$ & $0.39793-0.36149 i$ & $0.39793-0.36149 i$ \\
0.20 & $0.38459-0.43342 i$ & $0.38883-0.39777 i$ & $0.39300-0.36207 i$ & $0.39696-0.32633 i$ & $0.40050-0.29056 i$ \\
0.40 & $0.35498-0.51546 i$ & $0.36539-0.44015 i$ & $0.37598-0.36462 i$ & $0.38528-0.28888 i$ & $0.39152-0.21308 i$ \\
0.50 & $0.32915-0.56460 i$ & $0.34451-0.46621 i$ & $0.36044-0.36748 i$ & $0.37380-0.26837 i$ & $0.38051-0.16934 i$ \\
0.60 & $0.28965-0.62309 i$ & $0.31268-0.49797 i$ & $0.33704-0.37241 i$ & $0.35645-0.24626 i$ & $0.36232-0.12071 i$ \\
0.80 & $0.10472-0.79207 i$ & $0.16769-0.59291 i$ & $0.23613-0.39539 i$ & $0.28646-0.19715 i$ & $0.28191-0.00589 i$ \\
0.90 & $-0.18616-0.91652 i$ & $-0.05125-0.65940 i$ & $0.09557-0.41518 i$ & $0.20434-0.17202 i$ & $0.19003+0.05215 i$ \\
0.98 & $-1.2247-0.7046 i$ & $-0.77025-0.43494 i$ & $-0.31955-0.27607 i$ & $0.02188-0.13092 i$ & $0.04947+0.05337 i$ \\
\hline \hline
\end{tabular}

Sasaki-Nakamura, $s=-2, n=1$

\begin{tabular}{cccccc}
\multicolumn{5}{c}{ Sasaki-Nakamura, $s=-2, n=1$} \\
\hline$j$ & $m=-2$ & $m=-1$ & $m=0$ & $m=1$ & $m=2$ \\
\hline 0.00 & $2.2945-7.1873 i$ & $2.2945-7.1873 i$ & $2.2945-7.1873 i$ & $2.2945-7.1873 i$ & $2.2945-7.1873 i$ \\
0.20 & $2.5658-9.0390 i$ & $2.3970-8.0165 i$ & $2.2247-7.0665 i$ & $2.0424-6.1893 i$ & $1.8441-5.3859 i$ \\
0.40 & $2.543-10.849 i$ & $2.2599-8.6166 i$ & $1.9888-6.6818 i$ & $1.6731-5.0445 i$ & $1.2672-3.7148 i$ \\
0.50 & $2.344-11.695 i$ & $2.0427-8.7930 i$ & $1.7817-6.3679 i$ & $1.4444-4.4156 i$ & $0.9446-2.9579 i$ \\
0.60 & $1.933-12.458 i$ & $1.6641-8.8477 i$ & $1.4852-5.9476 i$ & $1.1812-3.7433 i$ & $0.6120-2.2745 i$ \\
0.80 & $-0.205-13.360 i$ & $-3.1529-8.2731 i$ & $0.4135-4.5896 i$ & $0.5162-2.2182 i$ & $0.0351-1.2045 i$ \\
0.90 & $-3.006-12.697 i$ & $-1.9831-7.0189 i$ & $-0.6196-3.3073 i$ & $0.0900-1.2838 i$ & $-0.04965-0.79665 i$ \\
0.98 & $-8.7106-7.3992 i$ & $-5.0880-2.5636 i$ & $-1.8011-0.6338 i$ & $-0.19532-0.23025 i$ & $0.07333-0.27876 i$ \\
\hline \hline
\end{tabular}

Teukolsky, $s=-2, n=1$

\begin{tabular}{llllll}
\hline 0.00 & $0.82322+0.75284 i$ & $0.82322+0.75284 i$ & $0.82322+0.75284 i$ & $0.82322+0.75284 i$ & $0.82322+0.75284 i$
\end{tabular} $\begin{array}{llllll}0.20 & 0.84911+0.70754 i & 0.82947+0.72453 i & 0.80991+0.74533 i & 0.79038+0.76996 i & 0.77073+0.79838 i\end{array}$ $\begin{array}{lllllll}0.40 & 0.85814+0.65292 i & 0.81337+0.67702 i & 0.76818+0.71734 i & 0.72213+0.77414 i & 0.67346+0.84688 i\end{array}$ $\begin{array}{llllll}0.50 & 0.85843+0.61654 i & 0.79766+0.64004 i & 0.73512+0.68991 i & 0.66983+0.76696 i & 0.59731+0.86954 i\end{array}$ $\begin{array}{llllll}0.60 & 0.85685+0.56852 i & 0.77713+0.58786 i & 0.69265+0.64679 i & 0.60121+0.74743 i & 0.49254+0.88500 i\end{array}$ $\begin{array}{lllllll}0.80 & 0.84873+0.39392 i & 0.72054+0.39027 i & 0.57393+0.46234 i & 0.39631+0.61795 i & 0.14457+0.81785 i\end{array}$ $\begin{array}{llllll}0.90 & 0.82922+0.19249 i & 0.66918+0.17074 i & 0.48666+0.24733 i & 0.25229+0.42940 i & -0.10621+0.60268 i\end{array}$ \begin{tabular}{lllllll}
0.98 & $0.65127-0.27305 i$ & $0.44118-0.27437 i$ & $0.27060-0.14913 i$ & $0.10617+0.07862 i$ & $-0.17382+0.14948 i$ \\
\hline \hline
\end{tabular}

TABLE VII: Values of the QNM frequencies and of the angular momentum parameter $\nu$ in the Schwarzschild limit.

\begin{tabular}{cccc}
$n$ & $s=0, l=2$ & $s=-1, l=1$ & $s=-2, l=2$ \\
\hline 0 & $1.64072+0.24770 i$ & $1.85364+0.20371 i$ & $1.72837+0.23396 i$ \\
1 & $-1.91021-0.44483 i$ & $-1.17974-0.29072 i$ & $-2.00310-0.37593 i$ \\
\hline \hline & & $M \omega$ \\
$n$ & $s=0, l=2$ & $s=-1, l=1$ & $s=-2, l=2$ \\
\hline 0 & $0.48364-0.09676 i$ & $0.24826-0.09249 i$ & $0.37367-0.08896 i$ \\
1 & $0.46385-0.29560 i$ & $0.21452-0.29367 i$ & $0.34671-0.27392 i$ \\
\hline \hline
\end{tabular}

